\title{
ON ZERO AND POLE SURFACES OF FUNCTIONS OF TWO COMPLEX VARIABLES $\left({ }^{(1)}\right.$
}

\author{
BY \\ STEFAN BERGMAN
}

1. Some problems arising in the study of value distribution of functions of two complex variables. One of the objectives of modern analysis consists in the generalization of methods of the theory of functions of one complex variable in such a way that the procedures in the revised form can be applied in other fields, in particular, in the theory of functions of several complex variables, in the theory of partial differential equations, in differential geometry, etc. In this way one can hope to obtain in time a unified theory of various chapters of analysis. The method of the kernel function is one of the tools of this kind. In particular, this method permits us to develop some chapters of the theory of analytic and meromorphic functions $f\left(z_{1}, \cdots, z_{n}\right)$ of the class $\mathcal{L}^{2}\left(\mathfrak{B}^{2 n}\right)$, various chapters in the theory of pseudo-conformal transformations (i.e., of transformations of the domains $\mathfrak{B}^{2 n}$ by $n$ analytic functions of $n$ complex variables) etc. On the other hand, it is of considerable interest to generalize other chapters of the theory of functions of one variable, at first to the case of several complex variables. In particular, the study of value distribution of entire and meromorphic functions represents a topic of great interest. Generalizing the classical results about the zeros of a polynomial, Hadamard and Borel established a connection between the value distribution of a function and its growth. A further step of basic importance has been made by Nevanlinna and Ahlfors, who showed not only that the results of Hadamard and Borel in a sharper form can be obtained by using potential-theoretical and topological methods, but found in this way important new relations, and opened a new field in the modern theory of functions.

As one passes from the theory of functions of one variable to the case of two and more variables $\left({ }^{2}\right)$, the question of the value distribution becomes more complex and many directions arise which can be considered as a generalization of the above mentioned chapter of the theory of functions of one variable. However, the formulation of the problems which can be answered, and generalization of methods used in one variable, is not immediate.

A function of two complex variables may vanish in a domain on a segment

Received by the editors July 6, 1953.

(1) This work has been done under a contract with the Office of Naval Research.

(2) In the present paper we are considering the case of two variables. We note that a generalization of methods used in the following to the case of $n$ variables, $n>2$, presents only technical difficulties. 
of a (two-dimensional) surface and there exists an infinite variety of independent functionals which we can associate with these segments. Hereby the behavior of different functionals reflects different properties of the segments. Further, a generalization of methods of Nevanlinna and Ahlfors to the theory of functions of two variables is not immediate, and the whole problem of connecting the theory of analytic functions with a conveniently chosen class of real functions for which a generalization of potential-theoretical procedures is possible requires a revision.

Various investigations in the theory of functions of several variables (e.g., arising in the theory of the kernel function) teach us that, in contrast to the situation in one variable, it is useful to classify domains of definition of functions of two complex variables. In particular, so-called domains with a distinguished boundary surface are of great interest; these are domains on whose (three-dimensional) boundary lies a (two-dimensional) distinguished boundary surface. In many instances, a generalization of deeper results in the theory of functions of one variable to two variables is possible in the case of these domains, whereby the distinguished boundary surface (and not the threedimensional boundary), plays the role of the boundary curve.

In these domains an analytic function of two complex variables assumes the maximum of its absolute value not only on the boundary of the domain, but even on the distinguished boundary surface. There exist a generalized Cauchy formula [B. 1, 2; W. 1. 2; So. 1] $\left(^{3}\right)$ and a development in terms of functions which are orthonormal when integrating along the distinguished boundary surface [B. 11]. In the case of domains with the distinguished boundary surface, these facts permit us to generalize a number of results of the theory of functions of one variable whose generalization is not possible in general domains. If, however, one attempts to generalize results obtained by the use of potential-theoretical methods, one realizes that the class of $B$-harmonic functions (i.e., real parts of functions of two complex variables) is too special, so that not necessarily to every (say, continuous) function defined on the distinguished boundary surface does there exist a function which is $B$-harmonic in the domain and which assumes on the distinguished boundary surface the prescribed values. This situation leads us to extend the class of $B$-harmonic functions in such a way that the extended class possesses all properties needed in various function-theoretical procedures, but is sufficiently large so that the boundary value problem with the values prescribed on the distinguished boundary surface always has a solution. These requirements lead us to introduce certain functions of the extended class which (in contrast to the case of one variable) depend in general upon the domain of definition.

Once the functions of the extended class are introduced, one can easily define Green's function of the extended class and derive various integral

(3) Numbers in brackets refer to the bibliography. As a rule the name of the author is replaced by the first letter. 
formulas of Green's type. It has been shown in [B. 3, 4, 5; B.-S. 2] that, using these formulas, one obtains certain functionals which can be considered as a direct generalization of such notions as a number of points $a_{\nu}$ for which $f\left(a_{\nu}\right)=a$ in a circle $|z|<r$, as $\sum_{\left|a_{\nu}\right| \leqq r}\left|a_{\nu}\right|^{-\lambda}$, etc., and that for these functionals one obtains bounds in terms of expressions characteristic for the growth of the functions on certain three-dimensional hypersurfaces. There arises, however, a difference in comparison with the two-dimensional case; namely, while a family of circles with increasing radii covers the whole plane, a one parameter family of two-dimensional manifolds forms a three-dimensional hypersurface; choosing different families we obtain different hypersurfaces on which the growth of the function is considered.

The continuation of investigations of this kind can proceed in different directions. At first one can use further types of domains with a distinguished boundary surface to obtain analogous formulas connecting functionals of similar type with the growth of the function on hypersurfaces of more complicated structure than those considered in [B. 4]. On the other hand, the variety of the functionals which one can associate with segments of a surface is richer than those which one can associate in the case of one variable with a set of points. Further, in connection with the theory of pseudo-conformal mapping where we consider a mapping of a domain by a pair of functions, it is of interest to associate functionals with a pair of functions.

In the present paper we introduce functionals of a new type, which are considered in special domains $\mathfrak{B}$. These domains are bounded by two segments of analytic hypersurfaces. One of these segments lies in the analytic hypersurface $\left[\left|z_{2}\right|=1\right]$, so that the family of analytic surfaces $z_{2}=$ const. plays a distinguished role for the structure of the domain under consideration. In domains of this kind (see for details $\$ 2$ ), we introduce functionals which are denoted by $\mathcal{X}_{k}(\mathbf{P}), k=1,2$, which we associate with a pair $\left(f_{1}, f_{2}\right)$ of meromorphic functions. These functionals are formed as follows: Let $w(z), w(0)$ $=0, w^{\prime}(0)>0$, be the function which maps a simply connected domain $\mathfrak{B}^{2}$ of the $z$ plane onto the unit circle. We denote

$$
\left(z, Z ; \mathfrak{B}^{2}\right) \equiv\left|\frac{w(z)-w(Z)}{1-\overline{w(Z)} w(z)}\right|,
$$

as the $\mathcal{C}\left(\mathfrak{B}^{2}\right)$-distance between $z$ and $Z\left({ }^{4}\right)$. For every $z_{2},\left|z_{2}\right| \leqq 1$, we form the following four products: $P_{1}\left(z_{2}\right)$ and $P_{2}\left(z_{2}\right)$ are the products of $\mathcal{C}\left(\mathfrak{B}^{2}\left(z_{2}\right)\right)$ distances between every zero of $f_{1} \equiv f_{1}\left(z_{1}, z_{2}\right)$ and every zero and pole of $f_{2}$, respectively, and $P_{3}\left(z_{2}\right)$ and $P_{4}\left(z_{2}\right)$ are analogous products with zeros of $f_{1}$ being replaced by poles of $f_{1}$. $\left(\mathfrak{B}^{2}\left(z_{2}^{0}\right)\right.$ means the intersections of the domain $\mathfrak{B}$ under consideration with the plane $z_{2}=z_{2}^{0}=$ const.)

We introduce further "exceptional points" $\left(z_{1}^{(\nu)}, z_{2}^{(\nu)}\right), \nu=1,2, \cdots$, of the

(4) (1) is connected in a very simple manner with the non-Euclidean distance, see p. 443). 
pair $\left(f_{1}, f_{2}\right)$. (These are intersection points of zero and pole surfaces of $f_{1}$ with zero and pole surfaces of $f_{2}$, and certain other points. See $\S 2$.) We cut out from the projection $\left|z_{2}\right|<1$ of $\mathfrak{B}$ on the $z_{2}$-plane the projections $\left|z_{2}-z_{2}^{(\nu)}\right|<\mathrm{P}$, $\mathrm{P}>0$ sufficiently small, and we denote by $\mathfrak{S}_{\mathrm{P}}^{2}$ the remaining part of the unit circle. The function $\mathscr{H}_{1}(\mathrm{P})$ is the average of the generalized Blaschke product $\left[P_{1}\left(z_{2}\right) P_{4}\left(z_{2}\right) / P_{2}\left(z_{2}\right) P_{3}\left(z_{2}\right)\right]$ over $\Im_{\mathrm{P}}^{2}$.

The second functional $\mathcal{X x}_{2}(\mathrm{P})$ is formed in a similar way. We obtain for the sum $\mathscr{X}_{1}(\mathrm{P})+\mathscr{X}_{2}(\mathrm{P})$ upper bounds in terms of quantities which are connected with the growth of the functions $\left(f_{1}, f_{2}\right)$ and certain quantities which we associate with exceptional points of the pair $\left(f_{1}, f_{2}\right)$.

In $\$ 7$ we show that the kernel function of the functions of the extended class normalized in the sense of a conveniently chosen metric is finite.

Thus, in [B. 4] and in the present paper, we derive bounds for functionals characterizing certain properties of segments of zero and pole surfaces of analytic functions of two variables. These results seem to indicate that, in analogy to the case of one variable, in the case of two variables after an extended class has been introduced one can also establish formulas of Green's type which then can be used in deriving bounds for functionals characterizing in this or other respects the behavior of zero and pole surfaces of one or of a pair of functions of two complex variables. In the domains with a distinguished boundary surface of more complicated structure, one obtains further relations using Morse's theory of critical points. [See B.6].

Notation. Manifolds as a rule are denoted by German characters, and the upper index indicates the dimension of the manifold. In the case of fourdimensional manifolds the index 4 is omitted. $\mathfrak{M}^{n}\left(z_{2}^{0}\right)$ means the intersection of $\mathfrak{M}^{n}$ with the plane $z_{2}=z_{2}^{0}=$ const. In operating with the sets we use the usual symbols $\mathrm{S}$ or + (sum set), - (difference set), $\cap$ (intersection), and so on. For instance, the sum set of a family of curves or domains $\mathfrak{B}^{n}\left(\zeta_{2}\right)$, $n=1$ or 2 , each of which lies in a different plane $z_{2}=\zeta_{2}$, where $\zeta_{2}$ varies along a curve or in domain $\mathfrak{A}^{m}, m \leqq 2$, will be denoted by $S_{z_{2} \in \mathfrak{T}^{m}} \mathfrak{B}^{n}\left(z_{2}\right)$ or by $\left[\mathrm{SB}^{n}\left(z_{2}\right), z_{2} \in \mathfrak{Y}^{m}\right]$. Arguments of the functions are abbreviated and omitted as much as possible. $z_{k}, k=1,2$, is used, as a rule, as a complex variable $x_{k}+i y_{k}$ and as the point $\left(x_{k}, y_{k}\right)$, as well.

A function, say $f$, is often considered:

$1^{\circ}$. in the domain $\mathfrak{B}=\mathrm{S}_{\left|z_{2}\right|<1} \mathfrak{B}^{2}\left(z_{2}\right)$, where $\mathfrak{B}^{2}\left(z_{2}\right)$ is the domain bounded by the curve $\mathfrak{b}^{1}\left(z_{2}\right)=\left[z_{1}=h\left(\lambda, z_{2}\right), 0 \leqq \lambda \leqq 2 \pi\right], h\left(0, z_{2}\right)=h\left(2 \pi, z_{2}\right)$;

$2^{\circ}$. in $\mathfrak{b}^{3}=S_{\left|z_{2}\right|<1} \mathfrak{b}^{1}\left(z_{2}\right)$ (b $\mathfrak{b}^{3}$ represents a part of the boundary of $\left.\mathfrak{B}\right)$; and

$3^{\circ}$. on the distinguished boundary surface $\mathfrak{D}^{2}=\mathbf{S}_{0 \leqq \phi_{2} \leqq 2 \pi} \mathfrak{b}^{1}\left(\exp \left(i \phi_{2}\right)\right)$.

As a rule, a function $f\left(z_{1}, z_{2}\right),\left(z_{1}, z_{2}\right) \in \mathfrak{B}$, when considered in $\mathfrak{b}^{3}$, as a function of $\lambda$ and $z_{2}$, i.e., $f\left[h\left(\lambda, z_{2}\right), z_{2}\right]$, will be denoted by $f^{*} \equiv f^{*}\left(\lambda, z_{2}\right)$, and when considered on $\mathfrak{D}^{2}$ as a function of $\lambda$ and $\phi_{2}$, i.e., $f\left[h\left(\lambda, \exp \left(i \phi_{2}\right)\right)\right.$, $\left.\exp \left(i \phi_{2}\right)\right]$, will be denoted by $\tilde{f}$. However, an exception is made in the case of the function $h\left(\lambda, z_{2}\right)$ which is defined originally as a function of $\left(\lambda, z_{2}\right)$ and only in 
very few cases is considered as a function of two complex variables $z_{1}, z_{2}$. We write then $h_{1}\left(z_{1}, z_{2}\right)$ so that $h_{1}\left(\exp (i \lambda), z_{2}\right) \equiv h\left(\lambda, z_{2}\right)$. We note that we write

$$
\tilde{h} \equiv \tilde{h}\left(\lambda, \phi_{2}\right) \text { for } h\left(\lambda, \exp \left(i \phi_{2}\right)\right) \text {. }
$$

Other minor abbreviations or conventions are explained in the body of the paper.

2. Domains with a distinguished boundary surface. Functions of the extended class. Let $h_{1}\left(\zeta, z_{2}\right)$ be an analytic function of two complex variables $\zeta, z_{2}$ defined in $\left[1-\epsilon \leqq|\zeta| \leqq 1+\epsilon,\left|z_{2}\right| \leqq 1\right], \epsilon>0$. We assume that $\tilde{h}$ $\equiv h\left[\lambda, \exp \left(i \phi_{2}\right)\right]$, where $h \equiv h\left(\lambda, z_{2}\right)=h_{1}(\exp (i \lambda), z)$, and $\tilde{h}_{\lambda} \equiv \partial \tilde{h} / \partial \lambda$ are continuously differentiable functions of $\phi_{2}$ and $\lambda$ and that

$$
0<H_{1} \leqq|\tilde{h}| \leqq H_{2}<\infty, \quad\left|\tilde{h}_{\lambda}\right| \leqq H_{3}
$$

where $H_{k}<\infty$ are conveniently chosen constants (Hypothesis 2.I). We assume further that for every fixed $z_{2},\left|z_{2}\right| \leqq 1$,

$$
\mathfrak{b}^{1}\left(z_{2}\right)=\left[z_{1}=h, 0 \leqq \lambda \leqq 2 \pi\right],
$$

is a smooth simple curve which bounds a simply-connected, schlicht domain $\mathfrak{B}^{2}\left(z_{2}\right)$ of the $z_{1}$-plane. It includes the origin $z_{1}=0$ in its interior (Hypothesis 2.II). Using the classical theorems about variable domains [C. 1,$2 ; \mathrm{R} .1]$ we conclude that $h$ and $h_{\lambda}$ are continuously differentiable functions of $x_{2}$ and $y_{i}$ and that the function $g\left(z_{1} ; z_{2}\right), g\left(0 ; z_{2}\right)=0, g^{\prime}\left(0 ; z_{2}\right)>0$, mapping $\mathfrak{B}^{2}\left(z_{2}\right)$ onto the unit circle is a continuously differentiable function of $x_{2}, y_{2}$.

In the present paper we consider a special class of domains

$$
\mathfrak{B}=\underset{\left|z_{2}\right|<1}{\mathrm{~S}} \mathfrak{B}^{2}\left(z_{2}\right) \equiv\left[z_{1} \in \mathfrak{B}^{2}\left(z_{2}\right),\left(\left|z_{2}\right|<1\right)\right] \text {. }
$$

As it can be easily shown (see [B.-S. 2]), the boundary of $\mathfrak{B}$ consists of two segments of analytic hypersurfaces

$$
\begin{aligned}
& \mathfrak{b}^{3}=\underset{\left|z_{2}\right| \leqq 1}{S} \mathfrak{b}^{1}\left(z_{2}\right) \equiv\left[z_{1} \in \mathfrak{b}^{1}\left(z_{2}\right),\left(\left|z_{2}\right| \leqq 1\right)\right], \\
& \mathfrak{a}^{3}=\underset{\left|z_{2}\right|=1}{S} \mathfrak{B}^{2}\left(z_{2}\right) \equiv\left[\left|z_{1}\right| \in \mathfrak{B}^{2}\left(z_{2}\right),\left(\left|z_{2}\right|=1\right)\right] .
\end{aligned}
$$

The intersection

$$
\mathfrak{D}^{2}=\mathfrak{a}^{3} \cap \mathfrak{b}^{3}=\underset{\left|z_{2}\right|=1}{S} \mathfrak{b}^{1}\left(z_{2}\right) \equiv\left[z_{1} \in \mathfrak{b}^{1}\left(z_{2}\right),\left(\left|z_{2}\right|=1\right)\right]
$$

of the two above segments forms the distinguished boundary surface of $\mathfrak{B}$.

Let $\mathfrak{S}^{2}(\lambda)$ and $\mathfrak{h}^{1}(\lambda)$ denote the segment of an analytic surface

$$
\mathfrak{S}^{2}(\lambda)=\left[z_{1}=h,\left|z_{2}\right| \leqq 1\right] \quad(0 \leqq \lambda \leqq 2 \pi)
$$

and the curve

$$
\mathfrak{h}^{1}(\lambda)=\left[z_{1}=h,\left|z_{2}\right|=1\right] \quad(0 \leqq \lambda \leqq 2 \pi),
$$


respectively. We note that we can also write

$$
\begin{aligned}
& \mathfrak{b}^{3}=\underset{\lambda=0}{2 \pi} \mathfrak{Q}^{2}(\lambda) \equiv\left[\left(z_{1}, z_{2}\right) \in \mathfrak{G}^{2}(\lambda),(0 \leqq \lambda \leqq 2 \pi)\right], \\
& \mathfrak{D}^{2}=\bigcup_{\lambda=0}^{2 \pi} \mathfrak{h}^{1}(\lambda) \equiv\left[\left(z_{1}, z_{2}\right) \in \mathfrak{h}^{1}(\lambda),(0 \leqq \lambda \leqq 2 \pi)\right] .
\end{aligned}
$$

Further we assume that $h\left(\lambda, z_{2}\right)$ satisfy the inequalities

$$
\left|\frac{h_{\lambda}}{h_{z_{2}}}\right| \leqq H<\infty, \quad\left|\frac{\partial}{\partial z_{2}}\left(\frac{h_{\lambda}}{h_{z_{2}}}\right)\right| \leqq G<\infty
$$

(Hypothesis 2.III).

REMARK: If $h_{z_{2}}$ vanishes (at some points or identically) in $\left|z_{2}\right| \leqq 1$, by the transformation

$$
z_{1}^{\dagger}=z_{1}+a z_{2}, \quad \stackrel{\dagger}{z_{2}}=z_{2},
$$

one can make $h^{\dagger}\left(\lambda, z_{2}\right)=h\left(\lambda, z_{2}\right)+a z_{2}$ satisfy the first of the conditions (11).

As mentioned in $\S 1$, for a continuous real function, defined on the distinguished boundary surface $\mathfrak{D}^{2}$, there does not necessarily exist a $B$-harmonic function defined in $\mathfrak{B}$, which possesses the prescribed boundary values on $\mathfrak{D}^{2}$. (See [B. 9].) In order to be able to apply potential-theoretical methods in every domain $\mathfrak{B}$, an "extended class of functions," $\mathcal{E}(\mathfrak{B})$, have been introduced in [B. 4, 6, 9; Bers 1]. However, in the papers mentioned above, only continuous boundary values on $\mathfrak{D}^{2}$ have been considered. For our purposes, the case is of interest where the boundary value function becomes infinite at finitely many points of $\mathfrak{D}^{2}$. In the following we shall describe the boundary values in which we are interested and define the functions of the extended class for this more general case.

According to (10), $\mathfrak{D}^{2}$ is a one-parameter family of curves $\mathfrak{h}^{1}(\lambda), 0 \leqq \lambda \leqq 2 \pi$. Each of these curves bounds a segment $\mathfrak{S}^{2}(\lambda)$ of the analytic surface $\left[z_{1}=h\right.$, $\lambda=$ const]. Let a real function $\tilde{t}$ be given on $\mathfrak{D}^{2}$ which is continuous, except at finitely many points, say

$$
\lambda=\lambda^{\kappa \nu}, \quad \phi_{2}=\phi_{2}^{\lambda \nu}, \quad \kappa=1,2, \cdots, \kappa_{1} ; \nu=1,2, \cdots, \nu_{1}\left(\kappa_{1}\right),
$$

where $\tilde{t}$ becomes $+\infty$ or $-\infty$.

A function $e \equiv e\left(z_{1}, z_{2}\right) \equiv e\left(z_{1}, z_{2} ; \tilde{t}\right)$ will be said to be a function of the extended class $\mathcal{E}(\mathfrak{B})$ which possesses the boundary values $\tilde{t}$ on $\mathfrak{D}^{2}$ if $e$ satisfies the following two conditions.

$1^{\circ} . e^{*}=e^{*}\left[\lambda, z_{2} ; \tilde{t}\right], 0 \leqq \lambda \leqq 2 \pi$, for every fixed $\lambda$ in $\left|z_{2}\right|<1$ is that harmonic function of $x_{2}, y_{2}$ which assumes for $\left|z_{2}\right|=1$ boundary values $\tilde{t}, \phi_{2}$ $=\operatorname{arc} z_{2}$. If $\left\{\lambda^{\kappa \nu}, \phi_{2}^{\kappa \nu}\right\}, \lambda^{\alpha \nu}=\lambda^{\nu}, \kappa=1,2, \cdots, \kappa_{1}$, are infinity points of $\tilde{t}\left(\lambda^{\nu}, \phi_{2}\right)$, then we assume that $\left[e^{*}\left(\lambda^{\nu}, z_{2}\right)-c_{k \nu} \log \left|z_{2}-\exp \left(i \phi_{2}^{k \nu}\right)\right|\right]$ remain 
bounded if we approach the point $z_{1}=\tilde{h}\left(\lambda^{\nu}, \phi_{2}^{k \nu}\right), z_{2}=\exp \left(i \phi^{k \nu}\right)$ moving in the surface $z_{1}=h\left(\lambda^{\nu}, z_{2}\right)$ and remaining in the domain whose projection on the $z_{2}$-plane is $-\pi / 2<-\alpha \leqq \operatorname{arc}\left[z_{2} \cdot \exp \left(-i \phi_{2}^{k \nu}\right)+1\right] \leqq \alpha<\pi / 2$. Here $c_{k v}$ are (positive or negative) integers.

$2^{\circ}$. By the requirement $1^{\circ}$ the function $e\left(z_{1}, z_{2} ; \tilde{t}\right) \in \mathcal{E}(\mathfrak{B})$ is defined in $\mathfrak{b}^{3}$, see (10). According to (4) the boundary curve $\mathfrak{b}^{1}\left(z_{2}\right)$ of $\mathfrak{B}^{2}\left(z_{2}\right)$ lies in $\mathfrak{b}^{3}$. Thus by $1^{\circ}, e^{*}$ is determined on the boundary curve of the domain $\mathfrak{B}^{2}\left(z_{2}\right)$. For every fixed $z_{2}$ the function $e$ is defined to be that harmonic function of $x_{1}, y_{1}$ which on $\mathfrak{b}^{1}\left(z_{2}\right)$ assumes the boundary value obtained above. As it can be easily shown, these values are for $\left|z_{2}\right|<1$ continuous. For $\left|z_{2}\right|=1$ they can have (finitely many) infinity points. If $\tilde{h}\left(\lambda^{\kappa \nu}, \phi_{2}^{\kappa \nu}\right), \phi_{2}^{\kappa \nu}=\phi_{2}^{\kappa}, \nu=1,2, \cdots, n_{\kappa}$, are infinity points which lie on $\mathfrak{b}^{1}\left(\exp \left(i \phi_{2}^{\kappa}\right)\right)$, then we assume that

$$
e\left(z_{1}, \exp \left(i \phi_{2}^{\kappa}\right)\right)-\sum_{\nu=1}^{n_{\kappa}} c_{\kappa \nu}^{*} \log \left|z_{1}-\hbar\left(\lambda^{\kappa \nu}, \phi_{2}^{\alpha}\right)\right|
$$

remains bounded if $z_{1}$ converges to $z_{1}=\tilde{h}\left(\lambda_{2}^{k \nu}, \phi_{2}^{k \nu}\right)$, along a path lying in the plane $z_{2}=\exp \left(i \phi_{2}^{k}\right)$ and remaining in $\mathfrak{B}^{2}\left(i \phi_{2}^{*}\right)$. Here $c_{\kappa \nu}^{*}$ are conveniently chosen (positive or negative) integers. Using the fact that a function $f$, belonging to $\mathcal{E}(\mathfrak{B})$ in every lamina $\left[z_{1}=h\left(\lambda, z_{2}\right),\left|z_{2}\right|<1\right]$, is a harmonic function of $x_{2}, y_{2}$, and $f$ in every $\left[z_{1} \in \mathfrak{B}^{2}\left(z_{2}\right)\right],\left|z_{2}\right| \leqq 1$, is a harmonic function of $x_{1}, y_{1}$, we can derive for $f$ a generalized Poisson formula (see [B. 11; B.-M. 1; Bers 1]). This formula yields a representation of $f$ when we approach a point $z_{1}$ $=\tilde{h}\left(\lambda^{\kappa \nu}, \phi_{2}^{\kappa \nu}\right), z_{2}=\exp \left(i \phi_{2}^{\kappa \nu}\right)$ of $\mathfrak{D}^{2}$ along a path in $\left[\operatorname{Re}\left[z_{1}-z_{2} h_{z_{2}}\left(\lambda^{k \nu}, \exp \left(i \phi_{2}^{\kappa \nu}\right)\right)\right.\right.$ $\left.\left.-\tilde{h}\left(\lambda^{\alpha \nu}, \phi_{2}^{\kappa \nu}\right)\right]<0\right] \times\left[\operatorname{Re}\left[z_{2} \exp \left(-i \phi_{2}^{\kappa \nu}\right)-1\right]<0\right]$.

As mentioned in $\$ 1$, the aim of the present paper is to give bounds for certain functionals $\mathscr{X}_{1}(\mathrm{P})$ and $\mathscr{X}_{2}(\mathrm{P})$ (see $\S 6$ ) of segments of zero and pole surfaces of a pair of functions $f_{1}$ and $f_{2}$ which are meromorphic in $\overline{\mathfrak{B}}$. These functionals are integrals over a domain $\mathfrak{S}_{\mathrm{P}}^{2}$. $\mathfrak{S}_{\mathrm{P}}^{2}$ is the projection of $\mathfrak{B}$ onto the $z_{2}$-plane (i.e. $\left[\left|z_{2}\right|<1\right]$ ) from which the projections $\left[\left|z_{2}-z_{2}^{(\nu)}\right|<\mathrm{P}\right]$ of the neighborhoods of exceptional points $\left(z_{1}^{(\nu)}, z_{2}^{(\nu)}\right)$ in $\mathfrak{B}$ of the pair $\left(f_{1}, f_{2}\right)$ are cut out.

These exceptional points consist of:

(a) Intersection points of the surfaces $\left[\log \left|f_{1}\right|= \pm \infty\right]$ and $\left[\log \left|f_{2}\right|\right.$ $= \pm \infty]$

(b) On different places, see 4.II.2, 4.III., etc. (see $\S \S 4$ and 5), we make assumptions about some properties of the functions $f_{1}$ and $f_{2}$; e.g., that $f_{2}, f_{1}$ or functions connected with them have at every point $\left(z_{1}, z_{2}\right)$ of $\mathfrak{B}$ or $\mathfrak{b}^{3}$ series developments whose first coefficients satisfy certain inequalities. In general, these hypotheses are fulfilled. But there can exist some points where these requirements are not fulfilled. These points $\left(z_{1}^{(\nu)}, z_{2}^{(\nu)}\right)$ belong also to the set of exceptional points. The projections of these exceptional points do not belong to $\widetilde{S}_{\mathrm{P}}^{2}$ (Hypothesis 2.IV). 
$f_{k}, k=1,2$, does not vanish or become infinite identically on a surface $z_{2}=c=$ const., $|c| \leqq 1$ (Hypothesis $2 . \mathrm{V}$ ).

We assume that there are only finitely many exceptional points ( $\mathrm{Hy}$ pothesis 2.VI).

\section{An integral formula for functions of the extended class.}

Definitions. 1. A function $P=P\left(z_{1}, z_{2}\right)$ will be said to be a function of the class $\mathscr{H}(\mathfrak{B})$, if $(P-\log |f|) \in \mathcal{E}(\mathfrak{B})$, where $f$ is a function of two complex variables which is meromorphic in $\overline{\mathfrak{B}}$.

2. If $z_{1}=\alpha\left(z_{2}\right)$ is a zero of $f=f\left(z_{1}, z_{2}\right)$, then sg $\left(\alpha\left(z_{2}\right)\right)=1$; if $\alpha\left(z_{2}\right)$ is a pole of $f$, then $\operatorname{sg}\left(\alpha\left(z_{2}\right)\right)=-1$.

Formulas of Green's type for harmonic functions, possessing in the domain logarithmic singularities, represent a valuable tool for the study of meromorphic functions of one complex variable. As has been mentioned in $\S 1$, in order to be able to apply potential-theoretical methods, and in particular to define some notions analogous to Green's function, we have to introduce functions of the class $\mathscr{H}(\mathscr{B})$ and to derive theorems of Green's type for these functions.

ThEOREM 3.1. Let $P_{k}=P_{k}\left(z_{1}, z_{2}\right), k=1,2$, be two different functions of the

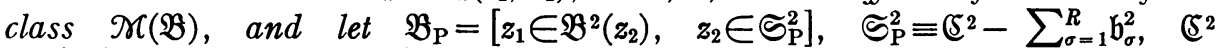
$=\left[\left|z_{2}\right|<1\right]$. Here $\mathfrak{b}_{\sigma}^{2}=\left|z_{2}-z_{2}^{(\sigma)}\right|<\mathrm{P}, z_{2}^{(\sigma)}$ being projections of exceptional points $(5)\left(z_{1}^{(\sigma)}, z_{2}^{(\sigma)}\right), \sigma=1,2, \cdots, \sigma_{0}<\infty$, of the pair $P_{k}, k=1,2$, in $\left[\left|z_{2}\right|<1\right]$. ( $\mathrm{P}$ is assumed to be sufficiently small.)

Let further $z_{1}=\alpha_{k \sigma} \equiv \alpha_{k \sigma}\left(z_{2}\right), \sigma=1,2, \cdots, N_{k}\left(z_{2}\right), N_{k}\left(z_{2}\right) \leqq N_{0}<\infty$, be the singularity surfaces of $P_{k}, k=1,2$. Then

$$
\begin{aligned}
\mathcal{F}_{\mathrm{P}}\left(P_{1}, P_{2}\right) & +\mathcal{B}_{\mathrm{P}}\left(P_{1}, P_{2}\right) \\
= & \frac{1}{4} \int_{\mathscr{q}^{2}} P_{1} P_{2} h_{z_{2}}^{-1} d z_{1} d z_{2}-\frac{\pi}{2} \int_{\mathscr{S}_{\mathrm{P}}{ }^{2}} \sum_{\nu} \operatorname{sg}\left(\alpha_{1 \nu}\left(z_{2}\right)\right) P_{2}\left(\alpha_{1 \nu}\left(z_{2}\right), z_{2}\right) d \omega_{2},
\end{aligned}
$$

where $\left(^{6}\right)$

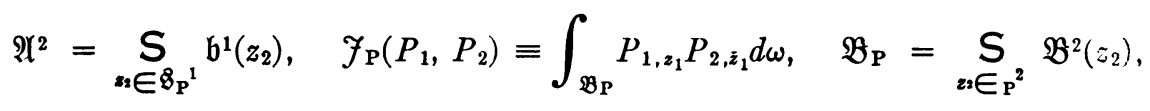

(2) $\mathcal{B}_{\mathrm{P}}\left(P_{1}, P_{2}\right) \equiv \frac{1}{2 i} \int_{\mathcal{S}_{\mathrm{P}}{ }^{2}} \int_{\lambda=0}^{2 \pi}\left[P_{2}^{*} p_{1} h_{\lambda} h_{z_{2}}^{-1}+P_{1}^{*} \frac{\partial\left(P_{2}^{*} h_{\lambda} h_{z_{2}}^{-1}\right)}{\partial z_{2}}\right] d \lambda d \omega_{2}$,

$$
\begin{aligned}
P_{k, z_{n}} & \equiv \frac{\partial P_{k}}{\partial z_{n}}, \quad k=1,2 ; \quad n=1,2, \quad p_{k}=\left(\frac{\partial P_{k}}{\partial z_{2}}\right)_{z_{1}=h\left(\lambda, z_{2}\right)}, \\
h_{\lambda} & =\frac{\partial h}{\partial \lambda}, \quad h_{z_{2}}=\frac{\partial h}{\partial z_{2}}, \quad d \omega_{k}=d x_{k} d y_{k}, \quad d \omega=d \omega_{1} d \omega_{2} .
\end{aligned}
$$

(5) See the end of $\S 2$.

(6) $\mathfrak{B}^{2}\left(z_{2}^{(0)}\right)=\mathfrak{B} \cap\left[z_{2}=z_{2}^{(0)}\right]$ have been defined on p. 415 . 
Here $\mathbb{B}_{\mathrm{P}}^{1}$ is the boundary of $\mathfrak{S}_{\mathrm{P}}^{2}, \mathfrak{b}^{1}\left(z_{2}\right)$ is the boundary of $\mathfrak{B}^{2}\left(z_{2}\right)$.

Proof. Let $z_{2} \in \Im_{\mathrm{P}}^{2}$ and let $\alpha_{k \nu}$ be the infinity points of $P_{k}, k=1,2$, in $\mathfrak{B}^{2}\left(z_{2}\right)$, while $\alpha_{k \mu}$ are infinity points on the boundary curve $\mathfrak{b}^{1}\left(z_{2}\right)$ of $\mathfrak{B}^{2}\left(z_{2}\right)$. By $\mathfrak{b}^{* 1}\left(z_{2}\right)$ we denote the boundary of the domain

$$
\mathfrak{B}^{* 2}=\mathfrak{B}^{2}\left(z_{2}\right)-\sum_{k=1}^{2} \sum_{\mu}\left[\left|z_{1}-\alpha_{k_{\mu}}\left(z_{2}\right)\right| \leqq \epsilon_{2}\right],
$$

and by $\mathfrak{b}^{* * 1}\left(z_{2}\right)$ the boundary of

$$
\begin{aligned}
\mathfrak{B}^{* * 2}\left(z_{2}\right)=\mathfrak{B}^{2}\left(z_{2}\right) & -\sum_{k=1}^{2}\left\{\sum_{\nu}\left[\left|z_{1}-\alpha_{k \nu}\left(z_{2}\right)\right| \leqq \epsilon_{1}\right]\right. \\
& \left.+\sum_{\mu}\left[\left|z_{1}-\alpha_{k \mu}\left(z_{2}\right)\right| \leqq \epsilon_{2}\right]\right\} .
\end{aligned}
$$

Here $\epsilon_{k}=\epsilon_{k}\left(z_{2}\right), k=1,2$, are chosen so small that all circles

$$
\left|z_{1}-\alpha_{k \nu}\left(z_{2}\right)\right|=\epsilon_{1} \text { and }\left|z_{1}-\alpha_{k \mu}\left(z_{2}\right)\right|=\epsilon_{2}
$$

have no intersection points with each other and that $\left|z_{1}-\alpha_{k v}\left(z_{2}\right)\right| \leqq \epsilon_{1}$ lies completely inside of $\mathfrak{B}^{2}\left(z_{2}\right)$. Applying (37b), p. 57 of [B. 8], we obtain

$$
\begin{aligned}
\int_{\mathfrak{B}^{* * 2}\left(z_{2}\right)} P_{1, z_{1}} P_{2, z_{1}} d \omega_{1}= & \frac{1}{2 i} \int_{\mathfrak{b}^{* 1}} P_{1, z_{1}} P_{2} d z_{1} \\
& -\frac{1}{2 i} \sum_{k=1}^{2} \sum_{\nu} \int_{\left|z_{1}-\alpha_{k \nu}\left(z_{2}\right)\right|=\varepsilon_{1}\left(z_{2}\right)} P_{1, z_{1}} P_{2} d z_{1} \\
& -\int P_{1, z_{1} \bar{z}_{1}} P_{2} d \omega_{1 .} .
\end{aligned}
$$

Here the last (double) integral in the right-hand side of (5) is taken over $\mathfrak{B}^{* * 2}\left(z_{2}\right)$. This last integral vanishes since $P_{1}$ is there a regular harmonic function, and therefore $P_{1, z_{1} z_{1}} \equiv \Delta P_{1 / 4}=0$ in $\mathfrak{B}^{* * 2}\left(z_{2}\right)$.

In the neighborhood of $\alpha_{1 v}\left(z_{2}\right), P_{1}=\mathrm{sg}\left(\alpha_{1 v}\left(z_{2}\right)\right) \log \left|z_{1}-\alpha_{1 v}\left(z_{2}\right)\right|+$ regular harmonic function; and

$$
\begin{aligned}
\frac{\partial \log \left|z_{1}-\alpha_{1 v}\left(z_{2}\right)\right|}{\partial z_{1}} & =\frac{1}{2}\left[\frac{\partial \log \left(z_{1}-\alpha_{1 v}\left(z_{2}\right)\right)}{\partial z_{1}}-\frac{\partial \log \left(\bar{z}_{1}-\overline{\left.\alpha_{1 v}\left(z_{2}\right)\right)}\right.}{\partial z_{1}}\right] \\
& =\frac{1}{2}\left(z_{1}-\alpha_{1 v}\left(z_{2}\right)\right)^{-1}=\frac{1}{2} r^{-1} e^{-i \psi}
\end{aligned}
$$

where $r e^{i \psi} \equiv z_{1}-\alpha_{1 v}\left(z_{2}\right)$. Since for $z_{2}=$ const. along the circles $\left|z_{1}-\alpha_{1 v}\left(z_{2}\right)\right|=r$, $d z_{1}=d\left(z_{1}-\alpha_{1 v}\left(z_{2}\right)\right)=i r e^{i \psi} d \psi$ we conclude in the usual way that

$$
\lim _{\sigma_{1} \rightarrow 0} \int_{\left|z_{1}-\alpha_{1 v}\left(z_{2}\right)\right|=\epsilon_{1}} P_{1, z_{1}} P_{2} d z_{1}=i \pi \cdot \operatorname{sg}\left(\alpha_{1 v}\left(z_{2}\right)\right) \cdot P_{2}\left(\alpha_{1 v}\left(z_{2}\right), z_{2}\right) .
$$


REMARK. We note that $P_{2}\left(\alpha_{1 v}\left(z_{2}\right), z_{2}\right), z_{2} \in \subseteq_{\mathrm{P}}^{2}$, is always finite since, according to the hypothesis $2 . \mathrm{IV}$, the projections on the $z_{2}$-plane of the intersection points of $\left[P_{1}= \pm \infty\right]$ and $\left[P_{2}= \pm \infty\right]$ do not belong to $\mathfrak{S}_{P^{2}}^{2}$.

Since $P_{1, z_{1}}$ is regular in $\left|z_{1}-\alpha_{2 v}\left(z_{2}\right)\right| \leqq \epsilon_{1}$ and $P_{2}$ is there logarithmically infinite, we conclude in an analogous way

$$
\lim _{\epsilon_{1} \rightarrow 0} \int_{\left|z_{1}-\alpha_{2_{\nu}}\left(z_{2}\right)\right|-\epsilon_{1}} P_{1, z_{1}} P_{2} d z_{1}=0 .
$$

Thus we finally obtain

$$
\begin{aligned}
\int_{\mathfrak{B}^{22^{2}}\left(z_{\mathrm{z}}\right)} P_{1, z_{1}} P_{2, z_{1}} d \omega_{1}= & \frac{1}{2 i} \int_{\mathfrak{b}^{* 1}\left(z_{2}\right)} P_{1, z_{1}} P_{2} d z_{1} \\
& -\frac{\pi}{2} \sum_{\nu} \operatorname{sg}\left(\alpha_{1 v}\left(z_{2}\right)\right) P_{2}\left(\alpha_{1 \nu}\left(z_{2}\right), z_{2}\right) .
\end{aligned}
$$

We proceed now to the study of the four-dimensional integrals and to the proof that the improper integrals $\epsilon_{2} \rightarrow 0$ can be replaced by ordinary ones. Obviously

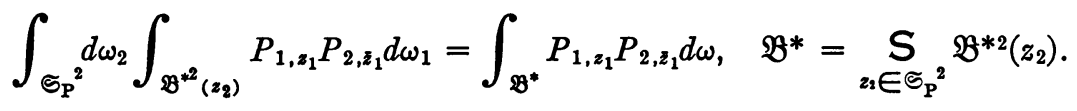

According to our assumptions the integrand is infinite, not higher than of first order on a two-dimensional manifold and therefore

$$
\lim _{\epsilon_{2} \rightarrow 0} \int_{\mathfrak{B}^{*}} P_{1, z_{1}} P_{2, z_{1}} d \omega=\int_{\mathfrak{B}} P_{1, z_{1}} P_{2, z_{1}} d \omega .
$$

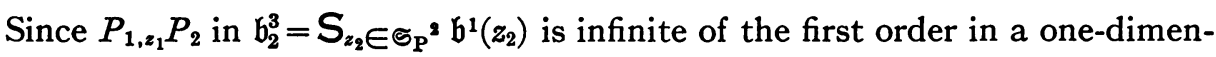
sional manifold,

$$
\begin{aligned}
\frac{1}{2 i} \lim _{\epsilon_{2} \rightarrow 0} \int_{\mathfrak{b}^{* 3}} P_{1, z_{1}} P_{2} d z_{1} d \omega_{2} & =\frac{1}{2 i} \int_{b_{2}^{3}} P_{1 . z_{1}} P_{2} d z_{1} d \omega_{2} \equiv J_{1}, \\
\mathfrak{b}^{* 3} & \equiv \underset{z_{2} \in \widetilde{S P}^{2}}{\operatorname{S}} \mathfrak{b}^{* 1}\left(z_{2}\right) .
\end{aligned}
$$

According to (2), see p. 416,

$$
P_{k}^{*} \equiv P_{k}\left[h\left(\lambda, z_{2}\right), z_{2}\right], p_{1} \equiv\left(\partial P_{1} / \partial z_{2}\right)_{z_{1}=h\left(\lambda, z_{2}\right)} .
$$

Thus $\partial P_{1}^{*} / \partial z_{2} \equiv\left(P_{1, z_{1}}\right) * h_{z_{2}}+p_{1}$ and therefore

$$
\left(P_{1, z_{1}}\right)^{*}=h_{z_{2}}^{-1}\left[\frac{\partial P_{1}^{*}}{\partial z_{2}}-p_{1}\right] \quad\left(\frac{\partial P_{1}^{*}}{\partial z_{2}} \equiv \frac{\partial P_{1}^{*}\left(\lambda, z_{2}\right)}{\partial z_{2}}\right),
$$

and 


$$
\begin{gathered}
J_{1}=J_{3}-J_{4}, \\
J_{3} \equiv \frac{1}{2 i} \int_{\left\{\varsigma_{\mathrm{P}}{ }^{2} \times \mathrm{t}^{1}\right\}} P_{2}^{*} \frac{\partial P_{1}^{*}}{\partial z_{2}} h_{\lambda} h_{z_{2}}^{-1} d \lambda d \omega_{2}, \quad J_{4} \equiv \frac{1}{2 i} \int_{\left\{\varsigma_{\mathrm{P}}{ }^{2} \times \mathfrak{t}^{1}\right\}} P_{2}^{*} p_{1} h_{\lambda} h_{z_{2}}^{-1} d \lambda d \omega_{2} \\
\mathrm{t}^{1}=[0 \leqq \lambda \leqq 2 \pi] .
\end{gathered}
$$

Let $z_{2}=\beta_{k \mu}(\lambda)$ denote the singularities of $P_{k}^{*}$ in $\Im_{\mathrm{P}}^{2}$, and $z_{2}=\beta_{k \nu}(\lambda)$ the singularities of $P_{k}^{*}$ on the boundary $\boldsymbol{g}_{\mathrm{P}}^{1}$ of $\mathfrak{S}_{\mathrm{P}}^{2}$. According to $2 . \mathrm{IVb}$, for fixed $\lambda$ the integrand of $J_{3}$ becomes (at the most at finitely many points) infinite at the most of the first order, and we can write (see (37a), p. 57 of [B. 8])

$$
\int_{\lambda=0}^{2 \pi} \int_{\varsigma_{\mathrm{P}}^{* * 2}} \frac{\partial P_{1}^{*}}{\partial z_{2}} \cdot P_{2}^{*} h_{\lambda} \cdot h_{z_{2}}^{-1} d \omega_{2} d \lambda=-\frac{1}{2 i} \int_{\lambda=0}^{2 \pi} \int_{\mathrm{BP}^{* 1}} P_{1}^{*} P_{2}^{*} h_{\lambda} h_{z_{2}}^{-1} d z_{2} d \lambda
$$

$$
\begin{aligned}
& +\frac{1}{2 i} \sum_{k=1}^{2} \int_{\lambda=0}^{2 \pi} \sum_{\nu} \int_{\left|z_{2}-\beta_{k \nu}(\lambda)\right|=\epsilon_{3}} P_{1}^{*} P_{2}^{*} h_{\lambda} h_{z_{2}}^{-1} d z_{2} d \lambda \\
& -\int_{\lambda=0}^{2 \pi} \int_{\varsigma_{\mathrm{P}}^{* * 2}} P_{1}^{*} \frac{\partial\left(P_{2}^{*} h_{\lambda} h_{z_{2}}^{-1}\right)}{\partial z_{2}} d \omega_{2} d \lambda,
\end{aligned}
$$

where

$$
\begin{aligned}
\mathfrak{S}_{\mathrm{P}}^{* * 2} \equiv \mathfrak{S}_{\mathrm{P}}^{* * 2}(\lambda) \equiv \mathfrak{S}_{\mathrm{P}}^{2} & -\sum_{k=1}^{2}\left\{\sum_{\nu}\left[\left|z_{2}-\beta_{k \nu}(\lambda)\right| \leqq \epsilon_{3}\right]\right. \\
& \left.+\sum_{\mu}\left[\left|z_{2}-\beta_{k \mu}(\lambda)\right| \leqq \epsilon_{4}\right]\right\}
\end{aligned}
$$

$8_{\mathrm{P}}^{* 1}$ denotes the boundary of $\Im_{\mathrm{P}}^{* 2} \equiv \Im_{\mathrm{P}}^{* 2}(\lambda) \equiv \Im_{\mathrm{P}}^{2}-\sum_{k=1}^{2} \quad \sum_{\mu}\left[\left|z_{2}-\beta_{k \mu}(\lambda)\right|\right.$ $\left.\leqq \epsilon_{4}\right]$. Here $\epsilon_{3} \equiv \epsilon_{3}(\lambda)$ and $\epsilon_{4} \equiv \epsilon_{4}(\lambda)$ are chosen so small that all circles $\left|z_{2}-\beta_{k \nu}(\lambda)\right|=\epsilon_{3}$ and $\left|z_{2}-\beta_{k \mu}(\lambda)\right|=\epsilon_{4}$ have no intersection points, and so that $\left|z_{2}-\beta_{k \nu}(\lambda)\right| \leqq \epsilon_{3}$ lie completely inside $\widetilde{\Im}_{\mathrm{P}}^{2}$.

Since $P_{1}^{*} P_{2}^{*}$ becomes at the most at finitely many points at the most logarithmically infinite, the second integral in the right-hand side of (14) vanishes if we go to limit $\epsilon_{3} \rightarrow 0$.

In the last integral on the right-hand side of (14) the integrand becomes infinite at the most of the first order along a one-dimensional manifold. We can therefore also pass to the limit and write

$$
\begin{aligned}
J_{3}= & \frac{1}{2 i} \int_{\lambda=0}^{2 \pi} \int_{\varsigma_{\mathrm{P}}^{2}} \frac{\partial P_{1}^{*}}{\partial z_{2}} P_{2}^{*} h_{\lambda} h_{z_{2}}^{-1} d \omega_{2} d \lambda=\frac{1}{4} \int_{\lambda=0}^{2 \pi} \int_{\mathrm{SP}^{1}} P_{1}^{*} P_{2}^{*} h_{\lambda} h_{z_{2}}^{-1} d z_{2} d \lambda \\
& -\frac{1}{2 i} \int_{\lambda=0}^{2 \pi} \int_{\varsigma_{\mathrm{P}}{ }^{2}} P_{1}^{*} \frac{\partial\left(P_{2}^{*} h_{\lambda} h_{z_{2}}^{-1}\right)}{\partial z_{2}} d \omega_{2} d \lambda .
\end{aligned}
$$

Combining (8), (10), (11), (13), and (15), we obtain (1). 
4. A bound for $\left|\mathcal{F}_{\mathrm{P}}\left(-\log \left|f_{1}\right|,-\log \left|f_{2}\right|+e_{2}\right)\right|$. Formula (3.1) establishes a relation between the sum of the integral $\mathcal{F}_{\mathrm{P}}\left(P_{1}, P_{2}\right)$ (over the domain $\mathfrak{B}_{\mathrm{P}}$ ) and the integral $\mathfrak{B}_{\mathrm{P}}\left(P_{1}, P_{2}\right)$ (over $\mathfrak{b}_{2}^{3}$ a part of $\mathfrak{b}^{3}$, see (2.9), p. 422) on one side, and the sum of certain integrals over the distinguished boundary surface, certain two-dimensional surfaces lying in $\mathfrak{b}^{3}$, and segments of zero and pole surfaces, on the other side. If we choose

$$
P_{1}=-\log \left|f_{1}\right|, \quad P_{2}=-\log \left|f_{2}\right|+e_{2}, \quad e_{2}=e\left(z_{1}, z_{2} ; \log \left|f_{2}\right|\right),
$$

see p. 418 , then, as we shall show in $\S 6$, the terms appearing on the righthand side of (3.1) can be interpreted as certain functionals associated with the segments of zero and pole surfaces of $f_{1}$ and $f_{2}$. (See for details $\$ 6$.)

In the present section bounds for

$$
\left|\mathcal{F}_{\mathrm{P}}\left(-\log \left|f_{1}\right|,-\log \left|f_{2}\right|+e_{2}\right)\right|,
$$

and in the next section bounds for

$$
\left|\mathcal{B}_{\mathrm{P}}\left(-\log \left|f_{1}\right|,-\log \left|f_{2}\right|+e_{2}\right)\right| \text {, }
$$

will be derived. Using (3.1), we obtain in this way bounds for the above mentioned functionals.

On the other hand, in order to be able to obtain bounds of this kind for $\left|\mathcal{F}_{\mathrm{P}}\left(-\log \left|f_{1}\right|,-\log \left|f_{2}\right|+e_{2}\right)\right|$, we have to make a number of hypotheses about the structure of the pole and zero surfaces of functions $f_{1}, f_{2}$. We proceed now to the formulation of these hypotheses.

Around every point

$$
\left[z_{1}=\alpha_{k \sigma}\left(z_{2}^{0}\right)\right]=\left[-\operatorname{sg}\left(\alpha_{k \sigma}\left(z_{2}^{0}\right) \log \left|f_{k}\left(z_{1}, z_{2}^{0}\right)\right|=\infty\right]\right.
$$

we draw the circle

$$
\mathfrak{夭}_{k \sigma}^{2}\left(z_{2}^{0}, \rho\right)=\left[\left|z_{1}-\alpha_{k \sigma}\left(z_{2}^{0}\right)\right|<\rho\right]
$$

and form around every branch of singularity surface

$$
\mathfrak{a}_{k \sigma}^{2}=\left[z_{1}=\alpha_{k \sigma}\left(z_{2}\right)\right], z_{2} \in S_{\mathrm{P}^{2}}
$$

a (four-dimensional) tube

$$
\mathrm{t}_{k \sigma}(\rho)=\underset{z_{2} \in \Im_{\mathrm{P}}{ }^{2}}{\mathrm{~S}} \mathbb{E}_{k \sigma}^{2}\left(z_{2}, \rho\right) .
$$

We assume that $\rho$ is chosen so small that the tubes $\mathrm{t}_{k \sigma}(\rho)$ have no intersection points $\left({ }^{7}\right)$.

HYPOTHESIS 4.I.

$$
\left|\left(z_{1}-\alpha_{k \sigma}\left(z_{2}\right)\right)^{\tau} f_{k}\right|^{-1} \leqq B_{k} \equiv B_{k}(\rho), \quad \tau=-\operatorname{sg}\left(\alpha_{k \sigma}\left(z_{2}\right)\right),
$$

(7) This is possible since the singularity surfaces of $\log \left|f_{1}\right|$ and $\log \left|f_{2}\right|$ have no intersection points in $\mathfrak{B}_{\mathrm{P}}$. 


$$
\left|\frac{\partial\left[\left(z_{1}-\alpha_{k \sigma}\left(z_{2}\right)\right)^{\tau} f_{k}\right]}{\partial z_{1}}\right| \leqq D_{k} \equiv D_{k}(\rho)
$$

hold for $\left(z_{1}, z_{2}\right) \in \mathrm{t}_{k \sigma}(\rho)$, and

$$
\begin{aligned}
\frac{1}{A_{k}} \leqq\left|f_{k}\right| \leqq A_{k}=A_{k}(\rho)<\infty, & A_{k}>1, \\
\left|\frac{\partial f_{k}}{\partial z_{1}}\right| & \leqq C_{k}=C_{k}(\rho)<\infty
\end{aligned}
$$

for $\left(z_{1}, z_{2}\right) \in \mathfrak{B}_{\mathrm{P}}-\sum_{\sigma} \mathrm{t}_{k \sigma}(\rho)$. Here $\rho, B_{k}, D_{k}, A_{k}, C_{k}$ are conveniently chosen constants.

Hypothesis 4.II. We assume that the zero and pole surfaces of $f_{k}, k$ $=1,2$, intersect the distinguished boundary surface $\mathfrak{D}^{2}$ at the most at finitely many points:

$$
\begin{array}{rr}
f_{2} \text { at } J_{\nu}=\left\{z_{1}^{(\nu)}, z_{2}^{(\nu)}\right\}, & \nu=1,2, \cdots, n, \\
f_{1} \text { at } J_{\nu}=\left\{z_{1}^{(\nu)}, z_{2}^{(\nu)}\right\}, & \nu=n+1, \cdots, n_{1},
\end{array}
$$

and we make the following hypotheses about the behavior of the functions $f_{k}, k=1,2$ in the neighborhood of the points $J_{\nu}$.

1. Through every $\left\{z_{1}^{(\nu)}, z_{2}^{(\nu)}\right\}, \nu=1,2, \cdots, n_{1}$, goes only one singularity surface of $\log \left|f_{k}\right|$, where either $k=1$ or 2 .

2. Let $z_{1}=\alpha_{2 \sigma}\left(z_{2}\right)$ be the equation of the singularity surface of log $\left|f_{2}\right|$ in the neighborhood of $J_{\nu}=\left\{z_{1}^{(\nu)}, z_{2}^{(\nu)}\right\}, \nu=1,2, \cdots, n$. Then we assume that in a sufficiently small neighborhood of $J_{\nu}$ the inequality $\left({ }^{8}\right)$

$$
\left|h\left(\lambda, z_{2}\right)-\alpha_{2 \sigma}\left(z_{2}\right)\right| \geqq a\left[\left|\lambda-\lambda^{(\nu)}\right|+\left|z_{2}-z_{2}^{(\nu)}\right|\right]
$$

holds where $0<a<1$.

Hyротнesis 4.III. We assume that the intersections $\left[f_{1}=0\right] \cap \mathfrak{b}^{3}$ and $\left[f_{1, z_{1}}=\infty\right] \cap \mathfrak{b}^{3}$ represent curves possessing the property that they can be divided into finitely many intervals $\mathfrak{i}_{\nu}^{1}$, such that in the neighborhood of each $i_{\nu}^{1}=\left[z_{1}=h\left(\lambda, Z_{2}(\lambda)\right), z_{2}=Z_{2}(\lambda)\right], Z_{2}(\lambda) \equiv Z_{2}^{(\nu)}(\lambda)$,

$$
f_{1}\left(h\left(\lambda, z_{2}\right), z_{2}\right) \equiv f_{1}^{*}=K_{1}(\lambda)\left(z_{2}-Z_{2}(\lambda)\right)+\cdots \text { and }\left|K_{1}(\lambda)\right| \geqq K>0 \text {. }
$$

Hypothesis 4.IV. In our further considerations we need auxiliary functions $s_{\nu}\left(z_{1}, z_{2}\right)$ which we associate with every intersection point $J_{\nu}$, $\nu=1,2, \cdots, n$, of singularities of $\log \left|f_{2}\right|$ with the distinguished boundary surface. So as to be able to form these functions $s_{\nu}$ we need some further additional hypotheses.

(8) The hypothesis 4 . II. 2 refers to the intersection of $\mathfrak{D}^{2}$ with singularities of $\log \left|f_{2}\right|$ only. 
Considering intersections of singularities of $\log \left|f_{2}\right|$ with the distinguished boundary surface, we distinguish two $\operatorname{cases}\left({ }^{9}\right)$.

Case 1.

$$
\begin{aligned}
& \alpha_{2 \sigma}\left(\exp \left(i \phi_{2}\right)\right) \in \mathfrak{B}^{2}\left(\exp \left(i \phi_{2}\right)\right) \text { for } \phi_{2}>\phi_{2}^{(v)}, \\
& \alpha_{2 \sigma}\left(\exp \left(i \phi_{2}\right)\right) \text { outside of } \mathfrak{B}^{2}\left(\exp \left(i \phi_{2}\right)\right) \text { for } \phi_{2}<\phi_{2}^{(\nu)} .
\end{aligned}
$$

Case 2. Where the opposite occurs, i.e., (14) holds for $\phi_{2}<\phi_{2}^{(\nu)}$, and (15) for $\phi_{2}>\phi_{2}^{(\nu)}$. Let

$$
z_{1}^{(\nu)}=h\left(\lambda_{\nu}, \exp \left(i \phi_{2}^{(\nu)}\right)\right), \quad z_{2}^{(\nu)}=\exp \left(i \phi_{2}^{(\nu)}\right) .
$$

In case 1 , the boundary values $\tilde{s}_{\nu} \equiv \bar{s}_{\nu}\left(\lambda, \phi_{2}\right)$ on $\mathfrak{D}^{2}$ are defined as follows:

$$
\begin{aligned}
\tilde{s}_{\nu}=\log \mid h\left(\lambda, \exp \left(i \phi_{2}\right)\right) & -\alpha_{2 \nu}\left(\exp \left(i \phi_{2}\right)\right) \mid \\
& \text { for } \lambda_{0} / 2>\left|\lambda-\lambda_{\nu}\right|, \Phi / 2 \geqq \phi_{2}-\phi_{2}^{(\nu)} \geqq 0 .
\end{aligned}
$$

For $\lambda_{0} \geqq\left|\lambda-\lambda_{\nu}\right| \geqq \lambda_{0} / 2,(\Phi / 2) \geqq \phi_{2}-\phi_{2}^{(\nu)} \geqq 0$ and for $\lambda_{0} \geqq\left|\lambda-\lambda_{\nu}\right|, \Phi \geqq \phi_{2}-\phi_{2}^{(\nu)}$ $>\phi / 2, \tilde{s}_{v}$ goes smoothly to zero, so that second derivatives with respect to $\lambda$ and to $\phi_{2}$ are continuous. These values are chosen in such a way that $\tilde{s}_{v}$ satisfies the inequality $s_{\nu}^{*} \geqq-\log a+\log \left|\lambda-\lambda_{\nu}\right|$, see (12) and p. 416. $\Phi$ is a conveniently chosen positive constant, and $\lambda_{0}<1$.

Everywhere else, i.e. for $\lambda_{0}>\left|\lambda-\lambda_{\nu}\right| \geqq 0,0>\phi_{2}-\phi_{2}^{(\nu)}$ or $\phi_{2}-\phi_{2}^{(\nu)}>\Phi, \tilde{s}_{\nu}=0$. At $\phi_{2}=\phi_{2}^{(\nu)}, \lambda_{0}>\left|\lambda-\lambda_{\nu}\right|, \tilde{s}_{\nu}$ has a jump.

In case $2, \tilde{s}_{\nu}$ is defined in an analogous manner with the only difference that in the definitions everywhere $\phi_{2}-\phi_{2}^{(\nu)}$ is replaced by $\phi_{2}^{(\nu)}-\phi_{2}$.

Let $s_{\nu}$ be the functions of the class $\mathcal{E}(\mathfrak{B})$ which assume the distinguished boundary surface values $\tilde{s}_{\nu}, \nu=1,2, \cdots, n$.

The function $\left(-s_{\nu}\right)$ is non-negative on the distinguished boundary surface and therefore also in $\mathfrak{B}$. At the point $J_{\boldsymbol{v}}$ it becomes infinite.

We assume that for conveniently chosen constants $p$ and $q$ there exist domains $\mathfrak{B}_{\nu}, \nu=1,2, \cdots, n, \mathfrak{B}_{\nu} \cap \mathfrak{B}_{\mu}=0$ for $\nu \neq \mu$, possessing the property that $\mathfrak{B}_{\nu} \supset\left(\mathfrak{B} \cap\left[-s_{\nu} \geqq p\right]\right)$ and such that the part of the boundary of $\mathfrak{B}_{\nu}$ which lies in $\mathfrak{b}^{3}$ includes $\left(\mathfrak{b}^{3} \cap\left[\left|\partial s_{\nu}^{*} / \partial z_{2}\right| \geqq q\right]\right)$. Further we assume that zero and pole surfaces of $f_{1}$ lie outside of the $\mathfrak{B}_{\nu}, \nu=1,2, \cdots, n$.

Hypothesis 4.V. After a function $s_{\nu} \in \mathcal{E}(\mathfrak{B})$ has been introduced for every intersection point $\left\{z_{1}^{(\nu)}, z_{2}^{(\nu)}\right\}, \nu=1,2, \cdots, n$, of $\mathfrak{D}^{2}$ with a singularity surface of $\log \left|f_{2}\left(z_{1}, z_{2}\right)\right|$, we define a further function $s \in \mathcal{E}(\mathfrak{B})$, which on the distinguished boundary surface assumes the values

$$
\begin{gathered}
\tilde{s}\left(\lambda, \phi_{2}\right)=\log \left|f_{2}\left(h\left(\lambda, \exp \left(i \phi_{2}\right)\right), \exp \left(i \phi_{2}\right)\right)\right|+\sum_{\nu=1}^{n} \tau_{\nu} \tilde{s}_{\nu}\left(\lambda, \phi_{2}\right), \\
\tau_{\nu}=-\operatorname{sg}\left[\alpha_{2 \nu}\left(\exp \left(i \phi_{2 \nu}\right)\right)\right] .
\end{gathered}
$$

${ }^{(9)}$ For simplicity's sake we assume that the case in which (14) holds for $\phi_{2}>\phi_{2}^{(\nu)}$ as well as for $\phi_{2}<\phi_{2}^{(\nu)}$ does not occur. In the following we shall consider, as a rule, only the case 1 . The modification of the formulas, when passing to the case 2 , are obvious. 
We assume that

$$
|s| \leqq S, \quad\left|\frac{\partial s}{\partial z_{1}}\right| \leqq T_{1} \quad \text { for }\left(z_{1}, z_{2}\right) \in \overline{\mathfrak{B}}
$$

where $S, T_{1}$ are conveniently chosen constants.

THEOREM 4.1. Let $f_{k} \equiv f_{k}\left(z_{1}, z_{2}\right), k=1,2$, be two analytic functions which are meromorphic in $\overline{\mathfrak{B}}$ and such that $P_{1}=-\log \left|f_{1}\right|$ and $P_{2}=-\log \left|f_{2}\right|+e_{2}$, $e_{2}=e\left(z_{1}, z_{2} ; \log \left|f_{2}\right|\right)$ satisfy the hypotheses of Theorem 3.1 .

Let

$$
z_{1}=\alpha_{k \sigma}\left(z_{2}\right), \quad k=1,2 ; \sigma=1,2, \cdots,
$$

be the equations of the singularities of $\log \left|f_{k}\left(z_{1}, z_{2}\right)\right|$.

We assume that for a conveniently chosen $\rho<1$ at every point of $\mathfrak{B}$, the hypotheses 4.I hold. Further, we assume that in the neighborhood of the intersection points $J_{\nu}$ of singularities of $\log \left|f_{k}\right|$ with the distinguished boundary surface $\mathfrak{D}^{2}$ the hypotheses 4.II-4.IV hold, and that the function $s$ introduced in $4 . \mathrm{V}$ satisfies the inequality (18). Then

$$
\begin{aligned}
& \left|\mathcal{F}_{\mathrm{P}}\left(-\log \left|f_{1}\right|,-\log \left|f_{2}\right|+e_{2}\right)\right| \\
& \leqq \frac{\pi}{2}\left[\sum_{k=1}^{2}\left(\rho+\frac{1}{2} B_{k} D_{k} \rho^{2}\right) \cdot A_{3-k} C_{3-k}\right] \cdot \sum_{k=1}^{2} S_{2}\left(\mathrm{~S}_{\sigma}^{2} \mathfrak{A}_{k \sigma}\right) \\
& \quad+\left[\pi T_{1}\left(\rho+\frac{1}{2} B_{1} D_{1} \rho^{2}\right)\right] \cdot \sum_{\sigma} \mathcal{C}\left(\mathfrak{A}_{1 \sigma}^{2}\right) \\
& \quad+\frac{1}{4} A_{1} C_{1}\left(A_{2} C_{2}+2 T_{1}\right) \cdot U(\mathfrak{B}) \\
& \quad+A_{1} C_{1} H_{3} \lambda_{0}\left(-\log a-\log \lambda_{0}+1\right) \cdot \sum_{\nu=1}^{n} \mathcal{S}_{1}\left(\mathfrak{B}_{v}\right) \\
& \quad+\pi n A_{1} C_{1} p P \lambda_{0}+2 \pi m n \lambda_{0} p P\left(\rho+\frac{1}{2} B_{1} D_{1} \rho^{2}\right)+\frac{\pi}{2} n p S_{2}\left(S_{\sigma} \mathfrak{A}_{1 \sigma}^{2}\right)
\end{aligned}
$$

where $A\left(\mathfrak{a}^{2}\right)$ denotes the area of $\mathfrak{a}^{2}, \mathcal{U}(\mathfrak{B})$ denotes the volume of $\mathfrak{B}$, and $\mathfrak{S}_{k}\left(\mathfrak{S S}^{m}\right)$, $k=1,2$, means the area of the projection $\mathrm{P}\left(\mathrm{SS}^{m}\right)$ of $\mathrm{(S}^{m}$, on the $z_{2}$-plane. Here $A_{k}$, $B_{k}, C_{k}, D_{k}$ have been introduced in (8)-(11), $H_{3}$ in 2.I, $a$ in (12), $T_{1}$ in 4.V, $\lambda_{0}$ and $p$ in 4.IV, $n$ in 4.II, $m$ is an upper bound for the number of segments of singularity lines

$$
z_{1}=h(\lambda, Z(\lambda)), \quad z_{2}=Z(\lambda)
$$

of $\log \left|f_{1}\right|$ in $\mathfrak{h}_{\nu}^{3}=S_{\lambda=\lambda_{\nu}-\lambda_{0}}^{\lambda_{\nu}+\lambda_{0}} \mathfrak{S}^{2}(\lambda)$ (see (2.7)) $P$ an upper bound for $\left|d z_{1} / d \lambda\right|$, and when we move along these lines. 
REMARK. $\delta_{1}\left(\oiint^{m}\right)$ means the area of the domain of $z_{2}$-plane consisting of all $z_{2}$ coordinates of the points $\left(z_{1}, z_{2}\right)$ of $\mathcal{S}^{m}$. $\delta_{2}\left(\mathfrak{R}^{2}\right)$ is the area of (in general multiply-sheeted) projection of $\mathfrak{N}^{2}$ on the $z_{2}$-plane. $\left(S_{2}\left(\mathfrak{R}^{2}\right)\right.$ is the sum of the areas of all sheets.)

Proof. Obviously

$$
\begin{aligned}
\mathcal{F}_{\mathbf{P}}\left(-\log \left|f_{1}\right|,-\log \left|f_{2}\right|+e_{2}\right)= & \mathcal{F}_{\mathbf{P}}\left(-\log \left|f_{1}\right|,-\log \left|f_{2}\right|+s\right) \\
& -\sum_{\nu=1}^{n} \tau_{\nu} \mathcal{F}_{\mathbf{P}}\left(-\log \left|f_{1}\right|, s_{\nu}\right)
\end{aligned}
$$

(see (17)) and we proceed to the derivation of upper bounds for each term on the right-hand side of (20) separately. We consider the first integral in a tube, say $t_{1 \sigma}(\rho)$. Using (5), (8), (9), (10), (11), (18) we obtain

$$
\begin{aligned}
& \int_{\overleftarrow{夭}_{1 \sigma^{2}\left(z_{2}, \rho\right) \cap \mathfrak{B}_{\left(z_{2}\right)}}}\left|\frac{\partial \log \left|f_{1}\right|}{\partial z_{1}} \cdot \frac{\partial\left(\log \left|f_{2}\right|-s\right)}{\partial \bar{z}_{1}}\right| d \omega_{1} \\
& \leqq \frac{1}{2} \int_{\mathfrak{C}_{1 \sigma}{ }^{2}\left(z_{2}, \rho\right) \cap \cap_{(}\left(z_{2}\right)^{2}}\left\{\left|z_{1}-\alpha_{1 \sigma}\left(z_{2}\right)\right|^{-1} \cdot\left|\frac{\partial\left(z_{1}-\alpha_{1 \sigma}\left(z_{2}\right)\right)}{\partial z_{1}}\right|\right. \\
& \left.+\left|\frac{\partial \log \left[\left(z_{1}-\alpha_{1 \sigma}\left(z_{2}\right)\right)^{\tau} f_{1}\right]}{\partial z_{1}}\right|\right\} \cdot\left|\frac{\partial \log \left|f_{2}\right|}{\partial \bar{z}_{1}}-\frac{\partial s}{\partial \bar{z}_{1}}\right| d \omega_{1} \\
& =\frac{1}{4} \int_{\rho^{*}=0}^{\rho} \int_{\phi_{2}=0}^{2 \pi}\left\{\frac{1}{\rho^{*}}+\left|\frac{\partial \log \left[\left(z_{1}-\alpha_{1 \sigma}\left(z_{2}\right)\right)^{\tau} f_{1}\right]}{\partial z_{1}}\right|\right\} \\
& \cdot\left|\frac{1}{\bar{f}_{2}} \frac{\partial \bar{f}_{2}}{\partial \bar{z}_{1}}-2 \frac{\partial s}{\partial \bar{z}_{1}}\right| d \omega_{1} \\
& \leqq \frac{1}{4} \int_{\rho^{*}=0}^{\rho} \int_{\phi_{2}=0}^{2 \pi}\left(\frac{1}{\rho^{*}}+B_{1} D_{1}\right)\left(A_{2} C_{2}+2 T_{1}\right) \rho^{*} d \rho^{*} d \phi_{2} \\
& =\frac{\pi}{2}\left(\rho+\frac{1}{2} B_{1} D_{1} \rho^{2}\right)\left(A_{2} C_{2}+2 T_{1}\right) \text {. }
\end{aligned}
$$

Thus

$$
\begin{aligned}
\left|\int_{\Sigma_{k=1}^{2} \Sigma_{\sigma} \mathrm{t}_{k \sigma}(\rho)} \frac{\partial \log \left|f_{1}\right|}{\partial z_{1}} \cdot \frac{\partial\left(\log \left|f_{2}\right|-s\right)}{\partial \bar{z}_{1}} d \omega\right| \\
\quad \leqq \frac{\pi}{2}\left[\sum_{k=1}^{2}\left(\rho+\frac{1}{2} B_{k} D_{k \rho^{2}}\right) A_{3-k} C_{3-k}\right] \cdot \sum_{k=1}^{2} \sum_{\sigma} \mathcal{A}\left(\mathfrak{A}_{k \sigma}^{2}\right) \\
\quad+\left[\pi T_{1}\left(\rho+\frac{1}{2} B_{1} D_{1} \rho^{2}\right)\right] \cdot \mathcal{S}_{2}\left(\mathrm{~S}_{\sigma}^{2} \mathfrak{A}_{1 \sigma}^{2}\right) .
\end{aligned}
$$

When considering $\mathcal{F}_{\mathbf{P}}\left(-\log \left|f_{1}\right|,-\log \left|f_{2}\right|+s\right)$ in $\mathfrak{B}_{\mathrm{P}}^{*}=\mathfrak{B}_{\mathrm{P}}-\sum_{k=1}^{2} \sum_{\sigma} \mathrm{t}_{k \sigma}(\rho)$ we obtain, using (10), (11), (18), 


$$
\begin{aligned}
&\left|\int_{\mathfrak{B}_{\mathrm{P}}^{*}} \frac{\partial \log \left|f_{1}\right|}{\partial z_{1}} \cdot \frac{\partial\left(\log \left|f_{2}\right|-s\right)}{\partial \bar{z}_{1}} d \omega\right| \\
& \qquad \int_{\mathfrak{B}_{\mathrm{P}}^{*}}\left|\frac{\partial \log \left|f_{1}\right|}{\partial z_{1}} \cdot \frac{\partial\left(\log \left|f_{2}\right|-s\right)}{\partial \bar{z}_{1}}\right| d \omega \\
& \quad=\int_{\mathfrak{B}_{\mathrm{P}}^{*}}\left|\frac{1}{2} \bar{f}_{1}^{-1} \frac{\partial f_{1}}{\partial z_{1}}\left(\frac{1}{2} \bar{f}_{2}^{-1} \frac{\partial \bar{f}_{2}}{\partial \bar{z}_{1}}-\frac{\partial s}{\partial \bar{z}_{1}}\right)\right| d \omega \\
& \leqq \frac{1}{4} A_{1} C_{1}\left(A_{2} C_{2}+2 T_{1}\right) \cdot \mathcal{V ( \mathfrak { B } ) .}
\end{aligned}
$$

We proceed similarly to determine a bound for $\left|\mathcal{F}_{\mathbf{P}}\left(-\log \left|f_{1}\right|, s_{\nu}\right)\right|$, but certain modifications are needed. They are caused by the fact that in the neighborhood of the intersection point $J_{\nu}\left\{z_{1}^{(\nu)}, z_{2}^{(\nu)}\right\}$ of the distinguished boundary surface with the surface $z_{1}=\alpha_{2 \sigma}\left(z_{2}\right), s_{\nu}$ becomes infinite.

We proceed now to determine the bounds for

$$
\left|\int_{\mathfrak{B}_{\mathrm{P}}} \frac{\partial \log \left|f_{1}\right|}{\partial z_{1}} \cdot \frac{\partial s_{\nu}}{\partial \bar{z}_{1}} d \omega\right| .
$$

(We choose the constants $\left({ }^{10}\right) p<\infty, q<\infty$, see 4 .IV, so large that log $\left|f_{1}\right|$ is regular in every $\mathfrak{B}_{\nu}$.)

According to (37b), page 57 of [B. 8], we have

$$
\begin{aligned}
\int_{\mathfrak{B}^{\dagger^{2}\left(z_{2}\right)}} \frac{\log \left|f_{1}\right|}{\partial z_{1}} \frac{\partial s_{\nu}}{\partial \bar{z}_{1}} d \omega_{1}= & \frac{1}{2 i} \int_{\mathfrak{b}^{1}\left(z_{2}\right)} \frac{\partial \log \left|f_{1}\right|}{\partial z_{1}} s_{\nu} d z_{1} \\
& -\frac{1}{2 i} \sum_{\sigma} \int_{\mathfrak{c}_{1 \sigma^{1}\left(z_{2}, p_{2}\right)}} \frac{\partial \log \left|f_{1}\right|}{\partial z_{1}} s_{\nu} d z_{1} .
\end{aligned}
$$

Here $\mathfrak{B}^{\dagger}\left(z_{2}\right)=\mathfrak{B}^{2}\left(z_{2}\right)-\sum_{\sigma} \mathfrak{G}_{1 \sigma}^{2}\left(z_{2}, \rho_{2}\right)$. Concerning $\mathfrak{C}_{1 \sigma}^{2}$ see $(5), \mathfrak{c}_{1 \sigma}^{1}$ are boundary curves of $\mathfrak{夭}_{1 \sigma}^{2}$. Since outside the interval $\left\{\lambda_{\nu}-\lambda_{0}, \lambda_{\nu}+\lambda_{0}\right\}, s_{\nu}$ vanishes on $\mathfrak{b}^{1}\left(z_{2}\right)$, we have for $z_{2} \in \mathcal{P}\left(\mathfrak{B}_{\nu}\right)$, see (16), (12), (2.1),

$$
\begin{aligned}
& \left|\frac{1}{2 i} \int_{\mathfrak{b}^{1}\left(z_{2}\right)} \frac{\partial \log \left|f_{1}\right|}{\partial z_{1}} s_{\nu} d z_{1}\right| \\
& \leqq \frac{1}{2} \int_{\lambda=\lambda_{\nu}-\lambda_{0}}^{\lambda_{\nu}+\lambda_{0}}\left|\frac{\partial \log \left|f_{1}\right|}{\partial z_{1}}\right||\log a+\log | \lambda-\lambda_{\nu}||\left|h_{\lambda}\left(\lambda, z_{2}\right)\right| d \lambda \\
& \left|\frac{1}{2 i} \int_{\mathcal{S}_{1}\left(\mathfrak{B}_{\nu}\right)} \int_{\mathfrak{b}^{1}\left(z_{2}\right)} \frac{\partial \log \left|f_{1}\right|}{\partial z_{1}} s_{\nu} d \omega_{2} d z_{1}\right| \\
& \quad \leqq A_{1} C_{1} H_{3} \lambda_{0}\left(-\log a-\log \lambda_{0}+1\right) \mathcal{S}_{1}\left(\mathfrak{B}_{\nu}\right) .
\end{aligned}
$$

(10) We note that the inequality $\left|\partial s_{\nu}{ }^{*} / \partial z_{2}\right|>q$ is used only in $\S 5$. 
We proceed now to the derivation of bounds for

$$
\begin{gathered}
\int_{\mathfrak{S}_{\mathrm{P}, \nu}^{2}} \int_{\mathfrak{b}^{1}\left(z_{2}\right)} \frac{\partial \log \left|f_{1}\right|}{\partial z_{1}} s_{\nu} d z_{1} d \omega_{2}=\frac{1}{2} \int_{\mathfrak{S}_{\mathrm{P}, \nu}^{2}} \int_{\mathfrak{b}^{1}\left(z_{2}\right)} \frac{f_{1, z_{1}}}{f_{1}} s_{\nu} d z_{1} d \omega_{z_{2}}, \\
\mathfrak{S}_{\mathrm{P}, \nu}^{2}=\mathfrak{S}_{\mathrm{P}}^{2}-\mathcal{S}_{1}\left(\mathfrak{B}_{\nu}\right) .
\end{gathered}
$$

According to the hypothesis 4.III, one can cover the neighborhood of $\mathfrak{b}^{3} \cap\left(\left[f_{1}=0\right]+\left[f_{1}=\infty\right]\right)$ in $\mathfrak{b}^{3}$ by finitely many tubes $\mathfrak{T}_{\nu \mu}^{3}$ each possessing the “axis" $\mathfrak{i}_{\nu}^{1}=\left[z_{1}=h\left(\lambda, Z_{2}(\lambda)\right), z_{2}=Z_{2}(\lambda)\right],-\lambda_{\nu} \leqq \lambda \leqq \lambda_{\nu+1}$. In each $\mathfrak{T}_{\nu \mu}^{3}$ we can represent $f_{1}$ in the form

$$
\begin{aligned}
\log \left|f_{1}\right| & =\log \left|\left(z_{1}-\alpha_{1 \sigma}\left(z_{2}\right)\right)^{-\tau}\right|+\log \left|\left(z_{1}-\alpha_{1 \sigma}\left(z_{2}\right)\right)^{\tau} f_{1}\right|, \\
\tau & \equiv-\operatorname{sg}\left(\alpha_{1 \sigma}\left(z_{2}\right)\right), \\
\frac{\partial \log \left|f_{1}\right|}{\partial z_{1}} & =\frac{1}{2}\left\{\frac{\partial \log \left(z_{1}-\alpha_{1 \sigma}\left(z_{2}\right)\right)^{-\tau}}{\partial z_{1}}+\frac{\frac{\partial}{\partial z_{1}}\left[\left(z_{1}-\alpha_{1 \sigma}\left(z_{2}\right)\right)^{\tau} f_{1}\right]}{\left(z_{1}-\alpha_{1 \sigma}\left(z_{2}\right)\right)^{\tau} f_{1}}\right\}, \\
\left|\frac{\partial \log \left|f_{1}\right|}{\partial z_{1}}\right| & \leqq \frac{1}{2}\left\{\frac{1}{\left|z_{1}-\alpha_{1 \sigma}\left(z_{2}\right)\right|}+B_{1} D_{1}\right\},
\end{aligned}
$$

by 4.I. Consequently, since according to $4 . \mathrm{IV}$ in the tube $\mathfrak{T}_{\nu \mu}^{3}$ with the axis $\mathfrak{i}_{\nu}^{1},\left|s_{\nu}\right| \leqq p$, and since $\left|d z_{1}\right| \leqq P d \lambda$,

$$
\begin{aligned}
\left|\int_{\mathfrak{T}_{\mu \nu}{ }^{3}} \frac{\partial \log \left|f_{1}\right|}{\partial z_{1}} s_{\nu} d \omega_{2} d z_{1}\right| & \\
& \equiv\left|\int_{\lambda=\lambda_{\nu}-\lambda_{0}}^{\lambda=\lambda_{\nu}+\lambda_{0}} \int_{\left|z_{2}-Z_{2}(\lambda)\right|<\rho} \frac{\partial \log \left|f_{1}\right|}{\partial z_{1}} s_{\nu} d \omega_{2} d z_{1}(\lambda)\right| \\
& \leqq \frac{1}{2}\left|\int_{\lambda_{\nu}-\lambda_{0}}^{\lambda_{\nu}+\lambda_{0}} \int_{0}^{2 \pi} \int_{0}^{\rho}\left[\frac{1}{\rho^{*}}+B_{1} D_{1}\right] p P \rho^{*} d \rho^{*} d \phi_{2} d \lambda\right| \\
& =2 \pi \lambda_{0} p P\left(\rho+\frac{1}{2} B_{1} D_{1} \rho^{2}\right) .
\end{aligned}
$$

Since we assumed that in $\mathcal{S}_{\lambda=-\lambda_{\nu}}^{\lambda_{\nu}+\lambda_{0}} \mathfrak{G}^{2}(\lambda)$ there are at the most $m$ tubes $\mathfrak{T}_{\nu \mu}^{3}$ and there are only $n$ points $\left\{z_{1}^{(\nu)}, z_{2}^{(\nu)}\right\}$, the sum of these integrals

$$
\sum_{\nu=1}^{n} \sum_{\mu}\left|\int_{\mathfrak{I}_{\nu \mu}{ }^{3}} \frac{\partial \log \left|f_{1}\right|}{\partial z_{1}} s_{\nu} d \omega_{2} d z_{1}\right| \leqq 2 \pi m n \lambda_{0} p P \cdot\left(\rho+\frac{1}{2} B_{1} D_{1} \rho^{2}\right) .
$$

On the remaining part of $\mathfrak{b}^{3}$ accordingly, (10), (11),

$$
\left|\frac{\partial \log \left|f_{1}\right|}{\partial z_{1}}\right|<\frac{1}{2} C_{1} A_{1}
$$


and therefore

$$
\left|\int_{\mathscr{A}_{\nu}{ }^{3}} \frac{\partial \log \left|f_{1}\right|}{\partial z_{1}} s_{\nu} d \omega_{2} d z_{1}\right| \leqq \int_{\lambda_{\nu}-\lambda_{0}}^{\lambda_{\nu}+\lambda_{0}} \frac{1}{2} A_{1} C_{1} p P \pi d \lambda=\pi A_{1} C_{1} p P \lambda_{0} .
$$

(The integration on the left-hand side is taken over $\mathfrak{A}_{\nu}^{3}=\mathfrak{b}^{3}-\mathscr{P}\left(\mathfrak{B}_{\nu}\right) \times \mathfrak{b}^{1}\left(z_{2}\right)$ $-\sum_{\nu=1}^{n} \sum_{\mu} \mathfrak{T}_{\nu \mu}^{3}$.) Since we have $n$ intersection points $\left\{z_{1}^{(\nu)}, z_{2}^{(\nu)}\right\}$,

$$
\sum_{\nu=1}^{n}\left|\int_{\mathscr{Q}_{\nu}{ }^{3}} \cdots\right| \leqq \pi n A_{1} C_{1} p P \lambda_{0} .
$$

It still remains to determine bounds for

$$
\left|\int_{\mathcal{S}_{\mathrm{P}}{ }^{2}}\left(\frac{1}{2} \int_{\mathrm{c}_{1}^{1}\left(z_{2}, \rho_{2}\right)}\left|\frac{\partial \log \left|f_{1}\right|}{\partial z_{1}}\right| s_{\nu} d z_{1}\right)\right| d \omega_{2}
$$

Now

$$
\lim _{\rho_{2} \rightarrow 0}\left|\frac{1}{2} \int_{\mathcal{c}_{1 \sigma}{ }^{1}\left(z_{2}, \rho_{2}\right)} \frac{\partial \log \left|f_{1}\right|}{\partial z_{1}} s_{\nu} d z_{1}\right|=\frac{\pi}{2} s_{\nu}\left(\alpha_{1 \sigma}\left(z_{2}\right), z_{2}\right)
$$

where $z_{1}=\alpha_{1 \sigma}\left(z_{2}^{0}\right)$ are infinity points of $-\operatorname{sg}\left(\alpha_{1 \sigma}\left(z_{2}^{0}\right)\right) \log \left|f_{1}\left(z_{1}, z_{2}^{0}\right)\right|$. But for $z_{2} \in \Phi\left(\mathfrak{B}_{\nu}\right)$ these points lie outside $\mathfrak{B}_{\nu}$, for $z_{2} \in \Im_{\mathrm{P}}^{2}-\mathcal{P}\left(\mathfrak{B}_{\nu}\right),\left|s_{\nu}\right| \leqq p$ on the boundary curve $\mathfrak{b}^{1}\left(z_{2}\right)$ of $\mathfrak{B}^{2}\left(z_{2}\right)$, and therefore also in $\mathfrak{B}^{2}\left(z_{2}\right)$. Consequently

$$
0 \leqq \frac{\pi}{2} s_{\nu}\left(\alpha_{1 \sigma}\left(z_{2}\right)\right) \leqq \frac{\pi}{2} p
$$

everywhere in $\mathfrak{B}$, so that

$$
\lim _{\rho_{2} \rightarrow 0}\left|\int_{\mathcal{S}_{\mathrm{P}}{ }^{2}} \sum_{\sigma}\left(\frac{1}{2} \int_{\mathfrak{c}_{1 \sigma}{ }^{1}\left(z_{2}, \rho_{2}\right)} \frac{\partial \log \left|f_{1}\right|}{\partial z_{1}} s_{\nu} d z_{1}\right) d \omega_{2}\right| \leqq \frac{\pi}{2} p S_{2}\left(\underset{\sigma}{S} \mathfrak{A}_{1 \sigma}^{2}\right)
$$

where $S_{2}\left(S_{\sigma} \mathfrak{R}_{1 \sigma}^{2}\right)$ is the area of the projections of the zero and the pole surface of $f_{1}$ on the $z_{2}$-plane.

Let us finally mention that the integrals

$$
\int_{\mathbb{C}_{1 \sigma^{2}\left(z_{2}, \rho\right)}} \frac{\partial \log \left|f_{1}\right|}{\partial z_{1}} \frac{\partial\left(\log \left|f_{2}\right|+s_{\nu}\right)}{\partial \bar{z}_{1}} d \omega_{2}
$$

tend to zero for $\rho \rightarrow 0$ since the integrand is infinite of the first order and the integration is taken over a two-dimensional domain whose area tends to zero.

5. A bound for $\mid B_{P}\left(-\log \left|f_{1}\right|,-\log \left|f_{2}\right|+e_{2} \mid\right.$. In determining an upper bound for the absolute value of the integral $\mathcal{B}_{P}\left(P_{1}, P_{2}\right)$ (over $\mathfrak{b}_{2}^{3}$ ), see (3.2), the line following (3.10), and (4.1), we write again $e_{2}=s+\sum_{\nu=1}^{n} s_{\nu}$ and shall determine a bound at first for $\left|\mathcal{B}_{P}\left(-\log \left|f_{1}\right|,-\log \left|f_{2}\right|+s\right)\right|$ and later for $\sum_{\nu=1}^{n}\left|\mathcal{B}_{P}\left(-\log \left|f_{1}\right|, s_{\nu}\right)\right|$.

In addition to the hypotheses made in $\$ \$ 3$ and 4 , we make now the following further assumptions. 
Hypothesis 5.I. In the part $\mathfrak{b}^{3}$ (see (2.4), (2.9), and p. 416) of the boundary of $\mathfrak{B}$

$$
\frac{\partial f_{2}^{*}}{\partial z_{2}}, \quad \log \left|f_{k}^{*}\right|, \quad k=1,2, \quad\left(\frac{\partial f_{1}}{\partial z_{2}}\right)_{z_{1}=h\left(\lambda, z_{2}\right)}
$$

become infinite along lines $z_{2}=\gamma_{2 \sigma}(\lambda), z_{2}=\beta_{k \sigma}(\lambda), k=1,2, z_{2}=\gamma_{1 \sigma}(\lambda)$, respectively. As before we assume that in a conveniently chosen (three-dimensional) tube $t^{3}$ with the above mentioned line as axis and intersection with $\mathfrak{S}^{2}(\lambda)$, see (2.7), being a circle of radius $\rho$,

$$
\begin{gathered}
\left|\left(z_{2}-\gamma_{2 \sigma}(\lambda)\right) \frac{\partial f_{2}^{*}}{\partial z_{2}}\right| \leqq \tau_{2}=z_{2}(\rho), \\
\frac{1}{Q_{k}} \leqq\left|\left(z_{2}-\beta_{k \sigma}(\lambda)\right)^{\tau} f_{k}^{*}\right| \leqq Q_{k} \equiv Q_{k}(\rho), \tau=-\operatorname{sg}\left(\beta_{k \sigma}(\lambda)\right), \\
\left|\left(z_{2}-\gamma_{1 \sigma}(\lambda)\right)\left(\frac{\partial f_{1}}{\partial z_{2}}\right)_{z_{1}=h\left(\lambda, z_{2}\right)}\right| \leqq L_{1} \equiv L_{1}(\rho)
\end{gathered}
$$

holds, while outside of the corresponding tube $t^{3}$ in $\mathfrak{b}^{3}$ the inequalities

$$
\begin{gathered}
\left|\frac{\partial f_{2}^{*}}{\partial z_{2}}\right| \leqq \tilde{c}_{4}=\tilde{c}_{4}(\rho), \\
\frac{1}{A_{k}} \leqq\left|f_{k}^{*}\right| \leqq A_{k}=A_{k}(\rho), \\
\left|\left(\frac{\partial f_{1}}{\partial z_{2}}\right)_{z_{1}=h\left(\lambda, z_{2}\right)}\right| \leqq \delta_{1}
\end{gathered}
$$

are valid.

Hypothesis 5.II. The functions $s_{\nu}$ introduced in $\S 4$, IV satisfy in $\mathfrak{n}_{\nu}^{3}$ : $\left[z_{1}=h\left(\lambda, z_{2}\right),\left|z_{2}-\delta\left(\lambda_{\nu}\right)\right|<\rho_{0}\left|\lambda-\lambda_{\nu}\right|<\lambda_{0}\right]$ the inequality

$$
\left|\frac{\partial s_{\nu}^{*}}{\partial z_{2}}\right| \leqq \frac{K}{\left|\lambda-\lambda_{\nu}\right|^{\mu}+\left|z_{2}-\delta\left(\lambda_{\nu}\right)\right|^{\eta}}, \quad \delta(\lambda)=\exp (i \lambda),
$$

where $K<\infty, \mu<\infty, \eta \leqq 2$ are conveniently chosen constants.

REMARK. Here we assume for simplicity's sake that $\delta(\lambda)=\exp (i \lambda)$, but we note that it would be possible to choose for $\delta(\lambda)$ some other function.

Hypothesis 5:III. For the function $s$ introduced in $\$ 4$, Hypothesis V, see (4.17), the inequality

$$
\left|\frac{\partial s\left(h\left(\lambda, z_{2}\right), z_{2}\right)}{\partial z_{2}}\right|=\left|\frac{\partial s^{*}}{\partial z_{2}}\right| \leqq T_{2}<\infty
$$

holds. 
Let $\mathfrak{j}_{k 0}^{1}, \mathfrak{j}_{k \infty}^{1}, k=1,2, \quad z_{2 \infty}^{1}, \quad \mathfrak{X}_{1 \infty}^{1}$ denote the respective intersections of $\left[\left|f_{k}\right|=0\right],\left[\left|f_{k}\right|=\infty\right],\left[\left|\partial f_{2}^{*} / \partial z_{2}\right|=\infty\right],\left[\left(\partial f_{1} / \partial z_{2}\right)_{z_{1}=h\left(\lambda, z_{1}\right)}=\infty\right]$ with $\mathfrak{b}_{2}^{3}$, see p. 422. Let further $i_{1}^{1}=i_{10}^{1}+i_{1 \infty}^{1}$.

Finally we assume that $p$ and $q$ have been chosen so large that $\mathbb{S}_{1 \infty}^{1}, \mathfrak{i}_{1}^{1}$ lie outside of the $\mathfrak{n}_{\nu}^{3}, \nu=1,2, \cdots, n$. See page 432 .

We remark also that we can assume that ${ }_{2 \infty}^{1}$ does not intersect the lines $\mathfrak{j}_{1}^{1}, \mathfrak{i}_{20}^{1}$, nor $\mathfrak{Z}_{1 \infty}^{1}$ intersect $\mathfrak{j}_{10}^{1}$, $\mathfrak{j}_{2}^{1}$ in $\mathfrak{b}^{3}$. (If necessary we can exclude these intersection points assuming that their projections belong to $\mathfrak{S}_{\mathrm{P}}^{2}$, see 2.IV.b.)

TheOREM 5.1. Let $f_{k}, k=1,2$, be two analytic functions which are meromorphic in $\mathfrak{B}$ and satisfy hypotheses of the Theorem 4.1. If in addition they satisfy the hypotheses 5.I-5.III, then

$$
\mathcal{B}_{\mathrm{P}}\left(-\log \left|f_{1}\right|,-\log \left|f_{2}\right|+e_{2}\right) \leqq B_{1}+B_{2}
$$

where

$$
\begin{aligned}
B_{1}= & \frac{\pi}{4}\left\{2 H\left|\log A_{1}\right| \rho\left[\left(\tilde{c}_{2} A_{2}+T_{2 \rho}\right) \mathcal{L}\left(3_{2 \infty}^{1}\right)+\left(\tilde{c}_{4} Q_{2}+T_{2 \rho}\right) \mathcal{L}\left(\dot{\mathfrak{i}}_{20}^{1}\right)\right]\right. \\
& +4\left(\left|\log A_{2}\right|+S\right) \rho H\left[A_{1} L_{1} \mathcal{L}\left(3_{1_{1 \infty}}^{1}\right)+\delta_{1} Q_{1} \mathcal{L}\left(\dot{\mathrm{i}}_{10}^{1}\right)\right] \\
& +\sum_{k=1}^{2}\left\{\frac{\pi}{4} \rho^{2}\left[H R_{k}+2 G\left(\left|\log A_{3-k}\right|+S(2-k)\right)\right]\right. \\
& \times\left[\frac{1}{2}-\log \rho+\left|\log Q_{k}\right|+S(k-1)\right] \mathcal{L}\left(\dot{\mathfrak{i}}_{k}^{1}\right) \\
& \left.+\frac{H}{4}\left[\left(\log \left|A_{k}\right|+(k-1) S\right) U_{k}\right] U\left(\mathfrak{b}^{3}\right)\right\} \\
R_{1}= & \left(A_{2} \tilde{c}_{4}+2 T_{2}\right), \quad R_{2}=\delta_{1} A_{1}, \quad U_{1}=\tilde{c}_{4} A_{2}+2 T_{2}, \\
U_{2}= & A_{1} \delta_{1}+2 G H^{-1}\left|\log A_{1}\right|, \quad \dot{i}_{k}^{1}=\dot{\mathfrak{i}}_{k 0}^{1}+\dot{\mathfrak{i}}_{k \infty}^{1},
\end{aligned}
$$

and $B_{2}$ is given in (34a). $\mathcal{L}\left(\mathrm{i}^{1}\right)$ denotes the "length" of the line segment $\mathrm{i}^{1}$, see (17).

Proof. I. In this part we derive an upper bound for $\mid B_{\mathbf{P}}\left(-\log \left|f_{1}\right|\right.$, $\left.-\log \left|f_{2}\right|+s\right) \mid$ while in II we shall compute a bound for $\left|\mathcal{B}_{\mathrm{P}}\left(-\log \left|f_{1}\right|, s_{v}\right)\right|$. According to (3.2)

$$
\mathcal{B}_{\mathrm{P}}\left(-\log \left|f_{1}\right|,-\log \left|f_{2}\right|+s\right)=\int_{0}^{2 \pi} \int_{\mathscr{S P}_{\mathrm{P}}{ }^{2}}\left(\tau_{1}+\tau_{2}+\tau_{3}\right) d \omega_{2} d \lambda,
$$

where

$$
\tau_{1}=\frac{1}{2 i}\left[\log \left|f_{1}^{*}\right| \frac{\partial\left(\log \left|f_{2}^{*}\right|-s^{*}\right)}{\partial z_{2}} \frac{h_{\lambda}}{h_{z_{2}}}\right]
$$




$$
\begin{aligned}
& \tau_{2}=\frac{1}{2 i}\left[\left(\frac{\partial \log \left|f_{1}\right|}{\partial z_{2}}\right)_{z_{1}=h\left(\lambda, z_{2}\right)}\left(\log \left|f_{2}^{*}\right|-s^{*}\right) \frac{h_{\lambda}}{h_{z_{2}}}\right], \\
& \tau_{3}=\frac{1}{2 i}\left[\log \left|f_{1}^{*}\right| \cdot\left(\log \left|f_{2}^{*}\right|-s^{*}\right) \frac{\partial}{\partial z_{2}}\left(\frac{h_{\lambda}}{h_{z_{2}}}\right)\right] .
\end{aligned}
$$

Since

$$
\frac{\partial \log \left|f_{2}^{*}\right|}{\partial z_{2}}=\frac{1}{2} \frac{1}{f_{2}^{*}} \frac{\partial f_{2}^{*}}{\partial z_{2}},
$$

$\tau_{1}$ becomes infinite along the curves $\dot{i}_{1}^{1} \equiv \dot{i}_{10}^{1}+\dot{i}_{1 \infty}^{1}, z_{2 \infty}^{1}$ and $j_{20}^{1}$. The second integrand $\tau_{2}$ becomes infinite along $\mathfrak{i}_{10}^{1}, \mathfrak{j}_{2}^{1} \equiv \dot{j}_{20}^{1}+\mathfrak{j}_{2 \infty}^{1} \equiv \mathfrak{j}_{20}^{1}+\left[\left|f_{2}^{*}\right|=\infty\right]$, and $\mathfrak{B}_{1 \infty}^{1}$. Finally, the third integrand $\tau_{3}$ becomes infinite along $\mathrm{i}_{k}^{1}, k=1,2$.

Around every point $\left(z_{1}^{0}, z_{2}^{0}\right), z_{1}^{0}=h\left(\lambda, z_{2}^{0}\right)$, of a singularity curve, say $\dot{i}^{1}$, we form in the lamina $\mathfrak{S}^{2}(\lambda)$ a $\operatorname{disc}\left[z_{1}=h\left(\lambda, z_{2}\right),\left|z_{2}-z_{2}^{0}\right|<\rho\right]=\delta^{2}\left(z_{1}^{0}, z_{2}^{0}\right)$ and form a (three-dimensional) tube

$$
\mathfrak{t}^{3}\left(\mathfrak{i}^{1}\right)=\underset{\left(z_{1}, z_{2}\right) \in \mathfrak{i}^{1}}{S} \mathfrak{d}^{2}\left(z_{1}, z_{2}\right) .
$$

The curve $\mathfrak{i}^{1}$ will be denoted as the axis of the tube. Determining bounds for the absolute value of the left side of (10), as before, we derive them separately at first in the tubes, and later in the remaining part of $\mathfrak{b}_{2}^{3}$. Considering $\tau_{1}$ in $\mathrm{t}^{3}\left(\mathrm{z}_{2 \infty}^{1}\right)$, see p. 433 , we write

$$
\begin{aligned}
& \tau_{1} \leqq \frac{1}{4}|\log | f_{1}^{*}||\left|\frac{1}{f_{2}^{*}} \cdot \frac{\partial f_{2}^{*}}{\partial z_{2}}-2 \frac{\partial s^{*}}{\partial z_{2}}\right| \cdot\left|\frac{h_{\lambda}}{h_{z_{2}}}\right| \\
& =\frac{1}{4}|\log | f_{1}^{*}||\left|\frac{\left(z_{2}-\gamma_{2 \sigma}(\lambda)\right)\left(\partial f_{2}^{*} / \partial z_{2}\right)}{\left(z_{2}-\gamma_{2 \sigma}(\lambda)\right) f_{2}^{*}}-2 \frac{\partial s^{*}}{\partial z_{2}}\right| \cdot\left|\frac{h_{\lambda}}{h_{z_{2}}}\right| \text {. }
\end{aligned}
$$

Thus by (4.10), (1), (4.18), (2.11), (7)

$$
\begin{aligned}
\left|\int_{\delta^{2}\left(z_{1}, z_{2}\right)} \tau_{1} d \omega_{2}\right| & \leqq \frac{1}{4}\left|\log A_{1}\right| H \int_{0}^{2 \pi} \int_{0}^{\rho}\left\{\frac{\tilde{c}_{2} A_{2}}{\rho^{*}}+2 T_{2}\right\} \rho^{*} d \rho^{*} d \phi_{2} \\
& =\frac{\pi}{2} H\left|\log A_{1}\right|\left(\tilde{c}_{2} A_{2} \rho+T_{2} \rho^{2}\right) .
\end{aligned}
$$

Consequently

$$
\left|\int_{z_{2 \infty}^{1}} d \lambda \int_{\delta^{2}\left(z_{1}, z_{2}\right)} \tau_{1} d \omega_{2}\right| \leqq \frac{\pi}{2} H\left|\log A_{1}\right|\left(\tilde{c}_{2} A_{2 \rho}+T_{2 \rho}^{2}\right) \mathcal{L}\left(z_{2 \infty}^{1}\right)
$$

where

$$
\mathcal{L}\left(z_{2 \infty}^{1}\right)=\int_{z_{2 \infty}^{1}} d \lambda
$$


In the case of $j_{20}^{1}$ we proceed exactly in the same way. Using (4.10), (2.11), (4), (2), (4.18), we obtain

$$
\begin{aligned}
\left|\int_{\mho^{2}\left(z_{1}, z_{2}\right)} \tau_{1} d \omega_{2}\right| & \leqq \frac{1}{4}\left|\log A_{1}\right| H \int_{0}^{2 \pi} \int_{0}^{\rho}\left\{\frac{\tilde{c}_{4} Q_{2}}{\rho^{*}}+2 T_{2}\right\} \rho^{*} d \rho^{*} d \phi_{2} \\
& =\frac{\pi}{2} H\left|\log A_{1}\right|\left(\tilde{\tau}_{4} Q_{2} \rho+T_{2} \rho^{2}\right)
\end{aligned}
$$

(19) $\left|\int_{\dot{i}_{20}{ }^{1}} \int_{b^{2}\left(z_{1}, z_{2}\right)} \tau_{1} d \omega_{2} d \lambda\right| \leqq \frac{\pi}{2} H\left|\log A_{1}\right|\left(\tilde{c}_{4} Q_{2} \rho+T_{2}{ }^{2}\right) \mathcal{L}\left(\dot{i}_{20}^{1}\right)$.

Considering $\tau_{1}$ in the tube $\mathbf{t}^{3}\left(\dot{\mathfrak{d}}_{1}^{1}\right)$ (around the zero and the pole lines of $f_{1}$ ) we use (2), (7), (4), (4.10), (2.11), (4.8) to obtain

$$
\begin{aligned}
& \left|\int_{\mathrm{b}^{2}\left(z_{1}, z_{2}\right)} \tau_{1} d \omega_{2}\right| \\
& \quad \leqq \frac{1}{4}\left(A_{2} \tilde{c}_{4}+2 T_{2}\right) H \int_{0}^{2 \pi} \int_{0}^{\rho}\left(\left|\log \rho^{*}\right|+\left|\log Q_{1}\right|\right) \rho^{*} d \rho^{*} d \phi_{2} \\
& \quad=\frac{\pi}{4} H \rho^{2}\left(A_{2} \tilde{c}_{4}+2 T_{2}\right)\left(\frac{1}{2}-\log \rho+\left|\log Q_{1}\right|\right),
\end{aligned}
$$

and therefore

$$
\begin{aligned}
\left|\int_{\mathrm{i}_{1}{ }^{1}} \int_{\mathrm{b}^{2}\left(z_{1}, z_{2}\right)} \tau_{1} d \omega_{2} d \lambda\right| & \\
& \leqq \frac{\pi}{4} H \rho^{2}\left(A_{2} \tilde{\tau}_{4}+2 T_{2}\right)\left(\frac{1}{2}-\log \rho+\left|\log Q_{1}\right|\right) \mathcal{L}\left(\dot{\mathrm{i}}_{1}^{1}\right) .
\end{aligned}
$$

Considering the next term $\tau_{2}$ in the tubes around singularities, we observe that by $5 . I$ in the tube $t^{3}\left(B_{1 \infty}^{1}\right)$

$$
\begin{aligned}
\left(\frac{\partial \log \left|f_{1}\right|}{\partial z_{2}}\right)=h\left(\lambda, z_{2}\right) z_{1} & =\frac{1}{2}\left(\frac{\partial f_{1}}{\partial z_{2}}\right)_{z_{1}=h\left(\lambda, z_{2}\right)} \cdot \frac{1}{f_{1}^{*}} \\
& =\frac{1}{2}\left\{\frac{1}{z_{2}-\gamma_{1 \sigma}(\lambda)}\left[\left(\frac{\partial f_{1}}{\partial z_{2}}\right)_{z_{1}=h\left(\lambda, z_{2}\right)}\left(z_{2}-\gamma_{1 \sigma}(\lambda)\right)\right]\right\} \frac{1}{f_{1}^{*}} .
\end{aligned}
$$

Thus by (3), (4.10), (4.18), (2.11)

$$
\begin{gathered}
\left|\tau_{2}\right| \leqq \frac{1}{2} \frac{L_{1}}{\rho^{*}} A_{1}\left(\left|\log A_{2}\right|+S\right) H \\
\left|\int_{B_{1 \omega^{1}}} \int_{b^{2}\left(z_{1}, z_{2}\right)} \tau_{2} d \omega_{2} d \lambda\right| \leqq \pi\left(\left|\log A_{2}\right|+S\right) H A_{1} L_{1} \rho \mathcal{L}\left(B_{1 \infty}^{1}\right) .
\end{gathered}
$$


Considering $\tau_{2}$ in $\mathrm{t}^{3}\left(\dot{\mathfrak{t}}_{10}^{1}\right)$ we have, according to $5 . \mathrm{I},(5),(2)$,

$$
\begin{aligned}
& \left|\left(\frac{\partial \log \left|f_{1}\right|}{\partial z_{2}}\right)_{z_{1}=h\left(\lambda, z_{2}\right)}\right| \\
& \quad=\frac{1}{2}\left|\left(\frac{\partial f_{1}}{\partial z_{2}}\right)_{z_{1}=h\left(\lambda, z_{2}\right)} \frac{\left(z_{2}-\beta_{1 \sigma}(\lambda)\right)^{-1}}{\left[\left(z_{2}-\beta_{1 \sigma}(\lambda)\right)^{-1} f_{1}^{*}\right]}\right| \leqq \frac{1}{2} \frac{\delta_{1} Q_{1}}{\rho^{*}}
\end{aligned}
$$

and therefore

$$
\begin{aligned}
& \left|\int_{\mathrm{i}_{10}{ }^{1}} \int_{\delta^{2}\left(z_{1}, z_{2}\right)} \tau_{2} d \omega_{2} d \lambda\right| \\
& \qquad \frac{1}{2}\left|\int_{\mathrm{i}_{10}{ }^{1}} \int_{0}^{2 \pi} \int_{0}^{\rho} \frac{\delta_{1} Q_{1}}{\rho^{*}}\left(\left|\log A_{2}\right|+S\right) H \rho^{*} d \rho^{*} d \omega_{2} d \lambda\right| \\
& =\pi \delta_{1} Q_{1}\left(\left|\log A_{2}\right|+S\right) H \rho \mathcal{L}\left(\dot{\mathrm{i}}_{10}^{1}\right) .
\end{aligned}
$$

Similarly in the tubes $t^{3}\left(j_{2}^{1}\right)$ around zero and pole surface $j_{2}^{1}$ using 5.I, (2) we have

$$
\begin{aligned}
|\log | f_{2}^{*}|| & =|\log |\left(z_{2}-\beta_{2 \sigma}(\lambda)\right)^{-\tau}\left(z_{2}-\beta_{2 \sigma}(\lambda)\right)^{\tau} f_{2}^{*}|| \\
& =|\log |\left(z_{2}-\beta_{2 \sigma}(\lambda)\right)^{\tau}|+\log |\left(z_{2}-\beta_{2 \sigma}(\lambda)\right)^{\tau} f_{2}^{*}|| \\
& \leqq-\log \rho^{*}+\left|\log Q_{2}\right|
\end{aligned}
$$

and therefore by (5), (4.10), (2.11), (4.18)

$$
\begin{aligned}
& \left|\int_{\mathrm{i}_{2}{ }^{1}} \int_{\delta^{2}\left(z_{1}, z_{2}\right)} \tau_{2} d \omega_{2} d \lambda\right| \\
& \quad \leqq \frac{1}{2}\left|\int_{\mathrm{i}_{2}{ }^{1}} \int_{0}^{2 \pi} \int_{0}^{\rho} \frac{\delta_{1} A_{1}}{2}\left(-\log \rho^{*}+\left|\log Q_{2}\right|+S\right) H \rho^{*} d \rho^{*} d \phi_{2} d \lambda\right| \\
& \quad=\frac{\pi}{4} \delta_{1} A_{1} H \rho^{2}\left(\frac{1}{2}-\log \rho+\left|\log Q_{2}\right|+S\right) \mathcal{L}\left(\dot{\mathrm{j}}_{2}{ }^{1}\right) .
\end{aligned}
$$

Finally, determining bounds for the integral of $\tau_{3}$ we note that in $\mathfrak{t}^{3}\left(\dot{\mathfrak{t}}_{k}^{1}\right)$ by 5.I and (2), $|\log | f_{k}^{*}|| \leqq-\log \rho^{*}+\left|\log Q_{k}\right|$ and therefore by (8), (4.10), (4.18), (2), (2.11)

$$
\begin{aligned}
&\left|\int_{\mathrm{i}_{1}{ }^{1}} \int_{\delta^{2}\left(z_{1}, z_{2}\right)} \tau_{3} d \omega_{2} d \lambda\right| \\
& \leqq \\
& \quad=\frac{\pi}{2} G\left(\left|\log A_{\mathrm{i}_{1}{ }^{1}} \int_{0}^{2 \pi} \int_{0}^{\rho} \frac{G}{2}\left(\left|\log A_{2}\right|+S\right)\left(-\log \rho^{*}+\left|\log Q_{1}\right|\right) \rho^{*} d \rho^{*} d \phi_{2} d \lambda\right|\right.
\end{aligned}
$$




$$
\begin{aligned}
& \left|\int_{\mathrm{i}_{2}{ }_{2}} \int_{\delta^{2}\left(z_{1}, z_{2}\right)} \tau_{3} d \omega_{2} d \lambda\right| \\
& \leqq \frac{\pi}{2} G \rho^{2}\left|\log A_{1}\right|\left(\frac{1}{2}-\log \rho+\left|\log Q_{2}\right|+S\right) \mathcal{L}\left(\dot{\mathrm{i}}_{2}^{1}\right) .
\end{aligned}
$$

Considering the integrands $\tau_{1}, \tau_{2}, \tau_{3}$ in the complementary parts of $\mathfrak{b}_{2}^{3}$ we have by (4.10), (4), (4.18), (2.11), (5), (7)

$$
\begin{aligned}
& \left|\tau_{1}\right| \leqq \frac{1}{4}\left|\log A_{1}\right|\left(\tilde{c}_{4} A_{2}+2 T_{2}\right) H, \\
& \left|\tau_{2}\right| \leqq \frac{1}{4} A_{1} \delta_{1}\left(\left|\log A_{2}\right|+S\right) H, \\
& \left|\tau_{3}\right| \leqq \frac{1}{2}\left|\log A_{1}\right|\left(\left|\log A_{2}\right|+S\right) G,
\end{aligned}
$$

so that the integral over the complementary parts

$$
\begin{aligned}
\mid \int \tau_{1} d \omega_{2} d \lambda & +\int \tau_{2} d \omega_{2} d \lambda+\int \tau_{3} d \omega_{2} d \lambda \mid \\
\leqq & \left\{\frac{1}{4}\left|\log A_{1}\right|\left(\tilde{c}_{4} A_{2}+2 T_{3}\right) H+\frac{1}{4} A_{1} \delta_{1}\left(\left|\log A_{2}\right|+S\right) H\right. \\
& \left.+\frac{1}{2}\left|\log A_{1}\right|\left(\left|\log A_{2}\right|+S\right) G\right\} \mho\left(\mathfrak{b}_{1}^{3}\right) .
\end{aligned}
$$

Combining (16), (19), (21), (24), (26), (28), (29), (30), and (34), we obtain $B_{1}$. II. In this part we shall show that

$$
\begin{aligned}
& \left|\sum_{\nu=1}^{n} B_{\mathrm{P}}\left(-\log \left|f_{1}\right|, s_{\nu}\right)\right| \\
& \leqq B_{2} \equiv \frac{\pi}{2} \rho^{2}\left(\frac{1}{2}-\log \rho+\left|\log Q_{1}\right|\right)\left(q H \sum_{\nu=1}^{n} \mathcal{L}_{\nu}\left(\dot{\mathrm{i}}_{1}^{1}\right)+p n G \mathcal{L}_{\left(\mathfrak{i}_{1}\right)}^{1}\right) \\
& \quad+\left(\frac{1}{2} A_{1} \delta_{1} H+\left|\log A_{1}\right| G\right) \lambda_{0}\left(-\log a-\log \lambda_{0}+1\right) \sum_{\nu=1}^{n} \mathcal{S}_{2}\left(\mathfrak{\Re}_{\nu}^{2}\right) \\
& \quad+\frac{\pi}{2}\left\{2 n\left|\log A_{1}\right| H K\left(\mu \lambda_{0}\left(1-\log \lambda_{0}\right)+\psi\right)+\sum_{\nu=1}^{n}\left(\rho p L_{1} A H \mathcal{L}_{\nu}\left(\mathfrak{S}_{1 \infty}^{1}\right)\right.\right. \\
& \left.\quad+\rho H \delta_{1} Q_{1} p \mathcal{L}_{\nu}\left(\mathfrak{i}_{10}^{1}\right)\right)+\frac{1}{2}\left[A_{1} \delta_{1} H_{p}+\left|\log A_{1}\right|(H q+G p)\right] \mathcal{U}\left(\mathfrak{h}_{\nu}^{3}\right)
\end{aligned}
$$

where $\psi$ is a constant for which the inequality

$$
2 \lambda_{0} \log \rho_{0} \leqq \psi \leqq\left(\lambda_{0} / 2\right) \log \left(\lambda_{0}^{\mu}+\rho_{0}^{2}\right)
$$


holds, and $\mathcal{L}_{y}\left(\mathfrak{i}^{1}\right)$ denotes the length of that part of $\dot{i}^{1}$ which lies in

$$
\mathfrak{h}_{\nu}^{3}=\underset{\lambda=\lambda_{\nu}-\lambda_{0}}{\lambda_{\nu}+\lambda_{0}} \mathfrak{S}^{2}(\lambda) .
$$

In order to prove (34a) we proceed to derive bounds for the absolute value of

$$
\begin{aligned}
\mathcal{B}_{\mathrm{P}}\left(-\log \left|f_{1}\right|, s_{\nu}\right) & =-\int_{\lambda=0}^{2 \pi} \int_{\mathcal{S P}_{\mathrm{P}}^{2}}\left(\tau_{4}^{(\nu)}+\tau_{5}^{(\nu)}+\tau_{6}^{(\nu)}\right) d \omega_{2} d \lambda, \\
\tau_{4}^{(\nu)} & =\frac{1}{2 i}\left(\frac{\partial \log \left|f_{1}\right|}{\partial z_{2}}\right)_{z_{1}=h\left(\lambda, z_{2}\right)} s_{\nu}^{*} \frac{h_{\lambda}}{h_{z_{2}}}, \\
\tau_{5}^{(\nu)} & =\frac{1}{2 i} \log \left|f_{1}^{*}\right| \frac{\partial s_{\nu}^{*}}{\partial z_{2}} \frac{h_{\lambda}}{h_{z_{2}}}, \\
\tau_{6}^{(\nu)} & =\frac{1}{2 i} \log \left|f_{1}^{*}\right| s_{\nu}^{*} \frac{\partial}{\partial z_{2}}\left(\frac{h_{\lambda}}{h_{z_{2}}}\right) .
\end{aligned}
$$

We repeat the same considerations as before. The only modification is that in this case instead of $\left(-\log \left|f_{2}\right|+s\right)$ the function $s_{\nu}$ appears which is regular in $\overline{\mathfrak{B}}$ except in a neighborhood of the point $\left\{z_{1}^{(\nu)}, z_{2}^{(\nu)}\right\}$ so that we have to derive separately bounds at first in the neighborhood $\mathfrak{n}_{\nu}^{3}$ of $\left\{z_{1}^{(\nu)}, z_{2}^{(\nu)}\right\}$ (see 5.II) and then in the complementary part $\mathfrak{b}_{2}^{3}-\mathfrak{n}_{\nu}^{3}$ of $\mathfrak{b}_{2}^{3}$. See p. 422 .

In the case of the term (36) we consider $\tau_{4}^{(\nu)}$ at first in $t^{3}\left(B_{1 \infty}^{1}\right), t^{3}\left(\mathfrak{i l l}_{10}^{1}\right), \mathfrak{n}_{v}^{3}$ and then in the complementary part.

Considering $\tau_{5}^{(\nu)}$ and $\tau_{6}^{(\nu)}$ we shall determine bounds in the tubes $\mathfrak{t}^{\mathbf{z}}\left(\mathfrak{i}_{1}^{1}\right)$ and $\mathfrak{n}_{\nu}^{3}$, and then in the complementary part $\mathfrak{b}_{2}^{3}-\mathfrak{t}^{3}\left(\mathfrak{l}_{1}^{1}\right)-\mathfrak{n}_{v}^{3}$.

We consider at first the term $\tau_{4}^{(v)}$ in $\mathfrak{t}^{3}\left(\mathfrak{B}_{1 \infty}^{1}\right)$ and $\mathfrak{t}^{3}\left(\dot{\mathfrak{d}}_{10}^{1}\right)$. See p. 433 and (13a). Using (22), (23), 4.II we obtain

$$
\begin{aligned}
\left|\int_{Z_{1 \omega^{1}}{ }^{1}} \int_{\delta^{2}\left(z_{1}, z_{2}\right)} \tau_{4}^{(p)} d \omega_{2} d \lambda\right| & \\
& \leqq \frac{1}{2} \int_{B_{10 \infty}{ }^{1}} \int_{0}^{2 \pi} \int_{0}^{\rho} \frac{1}{2} H \frac{L_{1}}{\rho^{*}} A_{1} S_{\nu}(\lambda) \rho^{*} d \rho^{*} d \phi_{2} d \lambda
\end{aligned}
$$

where $S_{\nu}(\lambda)=p$ for $\lambda_{\nu}-\lambda_{0} \leqq \lambda \leqq \lambda_{\nu}+\lambda_{0}$ and $S_{\nu}(\lambda)=0$ for the remaining values of $\lambda$. Consequently

$$
\left|\int_{B_{10}^{1}} \int_{b^{2}\left(z_{1}, z_{2}\right)} \tau_{4}^{(\nu)} d \omega_{2} d \lambda\right| \leqq \frac{\pi}{2} \rho p L_{1} A_{1} H \mathcal{L}_{\nu}\left(\mathcal{B}_{1 \infty}^{1}\right) .
$$

$\left(\mathcal{L}_{v}\left(\Re_{1 \infty}^{1}\right)\right.$ denotes the length of that part of $\mathfrak{B}_{1 \infty}^{1}$ which lies in $\mathfrak{H}_{v}^{3}$. See $\left.(34 \mathrm{~b})\right)$. Similarly, in $\mathrm{t}^{3}\left(\mathfrak{i}_{10}^{1}\right)$ using (25) we obtain 
$\left|\int_{\mathrm{i}_{10}{ }^{1}} \int_{\delta^{2}\left(z_{1}, z_{2}\right)} \tau_{4}^{(p)} d \omega_{2} d \lambda\right|$

$$
\begin{aligned}
& \leqq \frac{1}{2} \int_{\mathrm{i}_{10}{ }^{1}} \int_{0}^{2 \pi} \int_{0}^{\rho} \frac{1}{2} H \delta_{1} \frac{Q_{1}}{\rho^{*}} S_{\nu}(\lambda) \rho^{*} d \rho^{*} d \phi_{2} d \lambda \\
& \leqq \frac{\pi}{2} \rho H \delta_{1} Q_{1} p \mathcal{L}_{\nu}\left(\dot{\mathrm{i}}_{10}^{1}\right) .
\end{aligned}
$$

We proceed now to the determination of the bounds in $\mathfrak{n}_{v}^{3}$. See p. 432 .

In accordance with the hypothesis 5.III, $\mathfrak{t}^{\mathbf{8}}\left(\mathfrak{B}_{1 \infty}^{1}\right)$ and $\mathbf{t}^{3}\left(\dot{\mathrm{l}}_{10}^{1}\right)$ lie outside of $\mathfrak{n}_{\nu}^{3}$ so that by $(4.10)$, (5), we have

$$
\left|\frac{\partial \log \left|f_{1}\right|}{\partial z_{2}}\right|_{z_{1}=h\left(\lambda, z_{2}\right)} \leqq \frac{1}{2} A_{1} \delta_{1}
$$

for $\left(z_{1}, z_{2}\right) \in \mathfrak{n}_{\nu}^{3} \subset \mathfrak{b}_{2}^{3}-\mathrm{t}^{3}\left(B_{1 \infty}^{1}\right)-\mathfrak{t}^{3}\left(\mathfrak{d}_{10}^{1}\right)$.

According to (4.12) and the definition 4.IV.2

$$
\begin{aligned}
& -\tilde{s}_{\nu}\left(\lambda, \phi_{2}\right) \leqq-\log a-\log \left|\lambda-\lambda_{\nu}\right| \\
& \qquad \text { for }\left|\lambda-\lambda_{\nu}\right|<\lambda_{0}, 0<\phi_{2}-\phi_{2}^{(\nu)} \leqq \phi,
\end{aligned}
$$

$\tilde{s}_{\nu}\left(\lambda, \phi_{2}\right)=0$ on the remaining part of the distinguished boundary surface.

Since $s_{\nu}^{*}\left(\lambda, z_{2}\right)$ is for every fixed $\lambda, \lambda \neq \lambda$, an analytic function of a complex variable regular in $\left|z_{2}\right|<1$, it assumes the maximum value of its absolute value on the boundary and therefore in every $\mathfrak{S}^{2}(\lambda)$ and consequently in $\mathfrak{n}_{\nu}^{3} \cap \mathfrak{S}^{2}(\lambda)$

$$
\begin{gathered}
\left|\int_{\lambda=\lambda_{\nu}-\lambda_{0}}^{\lambda_{\nu}+\lambda_{0}} \int_{\mathfrak{n} \nu^{3} \cap \Phi^{2}(\lambda)} \tau_{\nu}^{*}\left(\lambda, z_{2}\right)\right| \leqq-\log a-\log \left|\lambda-\lambda_{\nu}\right| \\
\leqq-\frac{1}{4} \int_{\lambda=\lambda_{\nu}-\lambda_{0}}^{\lambda_{\nu}+\lambda_{0}} \int_{n_{\nu}{ }^{3} \cap \Phi^{2}(\lambda)} A_{1} \delta_{1}\left(\log a+\log \left|\lambda-\lambda_{\nu}\right|\right) H d \omega_{2} d \lambda \\
\leqq \frac{1}{2} A_{1} \delta_{1} H \lambda_{0}\left[-\log a-\log \lambda_{0}+1\right] \mathcal{S}_{1}\left(\mathfrak{n}_{\nu}^{3}\right)
\end{gathered}
$$

In the remaining part, $\mathfrak{h}_{v}^{3}-\mathfrak{n}_{v}^{3}$, we have by hypothesis $4 . \mathrm{IV}$ (see (4.16), (34b)) $\left|s_{\nu}\right| \leqq p$. Therefore by (42) and (2.11)

$$
\left|\int_{\mathfrak{b}_{2}{ }^{3}-\mathfrak{n}_{\nu}{ }^{8}} \tau_{4}^{(\nu)} d \omega_{2} d \lambda\right| \leqq \frac{1}{2} A_{1} \delta_{1} H p \mho\left(\mathfrak{h}_{\nu}{ }^{3}\right),
$$

where $\mathcal{U}\left(\mathfrak{h}_{v}^{3}\right)$ denotes the volume of $\mathfrak{h}_{v}^{3}$.

In deriving bounds for $\tau_{5}^{(v)}$ we observe at first that by (2), 4.IV (p. 426), (2.11) in $t^{3}\left(\mathfrak{l}_{1}^{1}\right)$ 


$$
\begin{aligned}
\left|\int_{\mathrm{i}_{1}{ }^{1}} \int_{\delta^{2}\left(z_{1}, z_{2}\right)} \tau_{5}^{(\nu)} d \omega_{2} d \lambda\right| \\
=\frac{1}{2}\left|\int_{\mathrm{i}_{1}{ }^{1}} \int_{\mathfrak{b}^{2}\left(z_{1}, z_{2}\right)} \log \right| f_{1}^{*}\left(\lambda, z_{2}\right)\left|\frac{\partial s_{\nu}^{*}\left(\lambda, z_{2}\right)}{\partial z_{2}} \frac{h_{\lambda}}{h_{z_{2}}} d \omega_{2} d \lambda\right| \\
\quad \leqq \frac{1}{2} \int_{\mathrm{i}_{1}{ }^{1}} \int_{0}^{2 \pi} \int_{0}^{\rho}\left(-\log \rho^{*}+\left|\log Q_{1}\right|\right) q H \rho^{*} d \rho^{*} d \phi_{2} d \lambda \\
=\frac{\pi}{2} q H \rho^{2}\left(\frac{1}{2}-\log \rho+\left|\log Q_{1}\right|\right) \mathcal{L}_{\nu}\left(\dot{\mathrm{i}}_{1}{ }^{1}\right)
\end{aligned}
$$

where $\mathcal{L}_{y}\left(\mathfrak{l}_{1}^{1}\right)$ denotes the length of that part of $\mathfrak{i}_{1}^{1}$ which lies in

$$
\mathfrak{h}_{\nu}^{3} \equiv \sum_{\lambda=\lambda_{\nu}-\lambda_{0}}^{\lambda_{\nu}+\lambda_{0}} \mathfrak{E}^{2}(\lambda)
$$

We proceed now to the derivation of the bounds in $\mathfrak{n}_{\nu}^{3}$, see p. 432. According to Hypothesis 5. II in $\mathfrak{n}_{\nu}^{3}$,

$$
\left|\frac{\partial s_{\nu}^{*}\left(\lambda, z_{2}\right)}{\partial z_{2}}\right| \leqq \frac{K}{\left|\lambda-\lambda_{\nu}\right|^{\mu}+\rho^{* 2}}
$$

where $K<\infty, \mu<\infty$. Therefore according to (2.11), (4.10)

$$
\left|\tau_{5}^{(\nu)}\right| \leqq \frac{1}{2}\left|\log A_{1}\right| H \frac{K}{\left|\lambda-\lambda_{\nu}\right|^{\mu}+\rho^{* 2}},
$$

and

$$
\begin{aligned}
\left|\int_{\mathfrak{n} \nu^{3}} \tau_{5}^{(\nu)} d \omega_{2} d \lambda\right| & \equiv\left|\int_{\lambda_{\nu}-\lambda_{0}}^{\lambda_{\nu}+\lambda_{0}} \int_{\mathfrak{N}_{\nu}{ }^{2}(\lambda)} \tau_{5}^{(\nu)} d \omega_{2} d \lambda\right| \\
& \leqq \frac{1}{2}\left|\log A_{1}\right| H K \int_{\lambda_{\nu}-\lambda_{0}}^{\lambda_{\nu}+\lambda_{0}} \int_{0}^{2 \pi} \int_{0}^{\rho_{0}} \frac{\rho^{*} d \rho^{*} d \phi_{2} d \lambda}{\left|\lambda-\lambda_{\nu}\right|^{\mu}+\rho^{* 2}} \\
& =\pi\left|\log A_{1}\right| H K\left[\mu \lambda_{0}\left(1-\log \lambda_{0}\right)+\psi\right]
\end{aligned}
$$

where $\psi=2 \int_{0}^{\lambda_{0}} d \lambda *\left[\log \left(\lambda^{* \mu}+\rho_{0}^{2}\right)\right]$ satisfies the inequality

$$
2 \lambda_{0} \log \rho_{0} \leqq \psi \leqq \frac{\lambda_{0}}{2} \log \left(\lambda_{0}^{\mu}+\rho_{0}^{2}\right) .
$$

In the remaining part, $\mathfrak{b}_{2}^{3}-\mathfrak{n}_{\nu}^{3}$, by $4 . \mathrm{IV},\left|\partial s_{\nu}^{*} / \partial z_{2}\right| \leqq q$ and therefore( $\left.{ }^{11}\right)$

$$
\left|\int_{\mathfrak{b}_{2}{ }^{3}-\mathfrak{t}^{3}\left(\mathbf{i}_{1}{ }^{1}\right)-\mathfrak{n}_{\nu}{ }^{3}} \tau_{5}^{(\nu)} d \omega_{2} d \lambda\right| \leqq \frac{1}{2}\left|\log A_{1}\right| H q \mathcal{U}\left(\mathfrak{h}_{\nu}{ }^{3}\right) .
$$

(11) We remind the reader that $s_{x}^{*}=0$ in $\mathfrak{b}_{2}^{8}-\mathfrak{h}_{\nu}^{8}$. 
Considering $\tau_{6}^{(\nu)}$ we proceed exactly in the same way as in the case of $\tau_{4}^{(\nu)}$, and using (2), (2.11), 4.IV we obtain in $t^{3}\left(\mathfrak{j}_{1}^{1}\right)$

$$
\begin{aligned}
\left|\int_{\left.t^{3} \dot{i}^{1}\right)} \tau_{6}^{(\nu)} d \omega_{2} d \lambda\right| & \leqq \frac{1}{2} \mid \int_{\left.t^{3} \dot{\alpha}^{1}\right)} G p\left(-\log \rho^{*}+\left|\log Q_{1}\right|\right) d \omega_{2} d \lambda \\
& \leqq \frac{\pi}{2} p G \rho^{2}\left[\frac{1}{2}-\log \rho+\left|\log Q_{1}\right|\right] \mathcal{L}\left(\dot{\mathrm{i}}_{1}^{1}\right),
\end{aligned}
$$

and in $\mathfrak{n}_{\nu}^{3}$ using (4.10), (43)

$$
\left|\int_{\lambda=\lambda_{\nu}-\lambda_{0}}^{\lambda_{\nu}+\lambda_{0}} \int_{n_{\nu}^{3} \cap \Phi^{2}(\lambda)} \tau_{6}^{(\nu)} d \omega_{2} d \lambda\right|
$$

$$
\leqq\left|\log A_{1}\right| G \lambda_{0}\left[-\log a-\log \lambda_{0}+1\right]_{2}\left(\delta \Re_{v}^{2}\right) .
$$

Finally, integrating in the remaining part, $\mathfrak{b}_{2}^{3}-\mathfrak{n}_{\nu}^{3}-\mathfrak{t}^{3}\left(\mathfrak{i}_{1}^{1}\right)$, we obtain

$$
\left|\int_{\mathfrak{b}_{2}^{3}-\mathfrak{t}^{3}\left(\dot{1}_{1}^{1}\right)-n_{\nu}{ }^{3}} \tau_{6}^{(v)} d \omega_{2} d \lambda\right| \leqq \frac{1}{2}\left|\log A_{1}\right| G p \mho\left(\mathfrak{h}_{v}^{3}\right) .
$$

Combining (40), (41), (45), (47), (49), (52), (53), (46), (51), and (54) we obtain $B_{2}$.

6. A geometric interpretation of terms on the right-hand side of (3.1). In $\S \S 4$ and 5 we derived upper bounds for the absolute value of $\mathcal{f}_{\mathbf{P}}\left(-\log \left|f_{1}\right|\right.$, $\left.-\log \left|f_{2}\right|+e_{2}\right)+\mathscr{B}_{\mathbf{P}}\left(-\log \left|f_{1}\right|,-\log \left|f_{2}\right|+e_{2}\right)$. According to (3.1), this last sum equals

$$
\begin{aligned}
& \frac{1}{4} \int_{\mathfrak{A}^{2}}\left(\log \left|f_{1}\right|\right)\left(\log \left|f_{2}\right|-e_{2}\right) h_{z_{2}}^{-1} d z_{1} d z_{2} \\
& \quad+\frac{\pi}{2} \int_{\Im_{\mathrm{P}}^{2}} \sum_{\nu=1}^{N_{1(22)}}\left\{\operatorname{sg}\left(\alpha_{1 v}\left(z_{2}\right)\right)\left[\log \left|f_{2}\left(\alpha_{1 v}\left(z_{2}\right), z_{2}\right)\right|-e_{2}\left(\alpha_{1 v}\left(z_{2}\right), z_{2}\right)\right]\right\} d \omega_{2},
\end{aligned}
$$

see p. 420 , so that the sum of expressions obtained in (4.19) and (5.8) (see also (5.9), (5.34a)) represents an upper bound for (1). In this section we shall give a geometrical interpretation of the terms appearing in (1). $P_{2} \equiv-\log \left|f_{2}\right|+e_{2}$ vanishes on the distinguished boundary surface, so that

$$
\int_{\mathscr{Q}^{2}} P_{1} P_{2} h_{z_{2}}^{-1} d z_{1} d z_{2}=\sum_{\nu} \int_{\mathscr{X}_{\nu}{ }^{2}}\left(\log \left|f_{1}\right|\right)\left(\log \left|f_{2}\right|-e_{2}\right) h_{z_{2}}^{-1} d z_{1} d z_{2}
$$

where $\mathfrak{A}_{\nu}^{2}=\left[\mathrm{Sb}^{1}\left(z_{2}\right), z_{2} \in \mathfrak{c}_{\nu}^{1}\right]$, see (2.2), are distinguished boundary surfaces of subdomains of $\mathfrak{B}$ which include the exceptional points $Q_{\boldsymbol{v}}$ of the pair $f_{1}, f_{2}$. Here $c_{\nu}^{1}=\left[\left|z_{2}-z_{2}^{(\nu)}\right|=\mathrm{P}\right]$ (see Hypothesis 2.IV, 4.II) are boundary curves of the domains $\mathfrak{b}_{\nu}^{2}$, see Theorem 3.1. The double integrals (2) can be expressed by lines integrals over the curves $\mathfrak{b}^{3}\left(z_{2}=z_{2}^{(\nu)}\right)$ and some additional terms. The 
behavior of these integrals, as we shall discuss elsewhere, depends to a large extent upon certain properties of the product $\log \left|f_{1}\right| \cdot\left(\log \left|f_{2}\right|-e_{2}\right)$ at exceptional points. (In particular one can derive for these integrals upper bounds, which depend essentially upon the local behavior of $f_{1}$ and $f_{2}$ in the neighborhood of exceptional points.)

We proceed now to the geometric interpretation of the second term in (1). We write

$$
\log \left|f_{2}\right|-e_{2}=\left[\log \left|f_{2}\right|-\varepsilon_{2}\right]+\left[\varepsilon_{2}-e_{2}\right] .
$$

Here $\varepsilon_{2}\left(z_{1}, z_{2}\right), z_{2}=$ const., is that harmonic function of $x_{1}, y_{1}$ which on the boundary curve $\mathfrak{b}^{1}\left(z_{2}\right)$ of $\mathfrak{B}^{2}\left(z_{2}\right)$ assumes the values

$$
\log \left|f_{2}\left(z_{1}, z_{2}\right)\right| \equiv \log \left|f_{2}^{*}\left(\lambda, z_{2}\right)\right| \text {. }
$$

The second term on the right-hand side of (3.1) will assume the form $\mathscr{X}_{1}(\mathrm{P})+\mathscr{X}_{2}(\mathrm{P})$ where

$$
\begin{aligned}
& \mathscr{X}_{1}(\mathrm{P})=\frac{\pi}{2} \int_{\mathfrak{S P}^{2}} \sum_{\nu=1}^{N_{1}\left(z_{2}\right)}\left\{\operatorname { s g } ( \alpha _ { 1 v } ( z _ { 2 } ) ) \left[\log \left|f_{2}\left(\alpha_{1 v}\left(z_{2}\right), z_{2}\right)\right|\right.\right. \\
& \left.\left.-\varepsilon_{2}\left(\alpha_{1 \nu}\left(z_{2}\right), z_{2}\right)\right]\right\} d \omega_{2}, \\
& \mathscr{H}_{2}(\mathrm{P})=\frac{\pi}{2} \int_{\widetilde{S P}^{2}} \sum_{v=1}^{N_{1\left(z_{2}\right)}}\left\{\mathrm{sg}\left(\alpha_{1 v}\left(z_{2}\right)\right)\left[\varepsilon_{2}\left(\alpha_{1 v}\left(z_{2}\right), z_{2}\right)-e_{2}\left(\alpha_{1 v}\left(z_{2}\right) ; z_{2}\right)\right]\right\} d \omega_{2} .
\end{aligned}
$$

If $z_{1}=\alpha_{2 n}\left(z_{2}\right)$ are the zeros and the poles of $f_{2}\left(z_{1}, z_{2}\right)$ in $\mathfrak{B}^{2}\left(z_{2}\right)$ and $g\left(z_{1}, Z_{1}, z_{2}\right)$ is the Green's function of $\mathfrak{B}^{2}\left(z_{2}\right)$

$$
\begin{aligned}
\log \left|f_{2}\left(z_{1}, z_{2}\right)\right|-\varepsilon_{2}\left(z_{1}, z_{2}\right) & =-\sum \operatorname{sg}\left(\alpha_{2 n}\left(z_{2}\right)\right) g\left(z_{1}, \alpha_{2 n}\left(z_{2}\right) ; z_{2}\right) \\
& \equiv \sum_{n=1}^{N_{2}\left(z_{2}\right)} \operatorname{sg}\left(\alpha_{2 n}\left(z_{2}\right)\right) \log \left(z_{1}, \alpha_{2 n}\left(z_{2}\right) ; \mathfrak{B}^{2}\left(z_{2}\right)\right) .
\end{aligned}
$$

Here

$$
\left(z_{1}, Z_{1} ; \mathfrak{B}^{2}\left(z_{2}\right)\right)=\left|\frac{w\left(z_{1} ; z_{2}\right)-w\left(Z_{1} ; z_{2}\right)}{1-\overline{w\left(Z_{1} ; z_{2}\right)} w\left(z_{1} ; z_{2}\right)}\right|
$$

and

$$
w\left(z_{1} ; z_{2}\right)=w\left(z_{1} ; z_{2}, \bar{z}_{2}\right), \quad w\left(0 ; z_{2}\right)=0,\left.\quad \frac{d w\left(z_{1} ; z_{2}\right)}{d z_{1}}\right|_{z_{1}=0}>0,
$$

denotes that analytic function which maps $\mathfrak{B}^{2}\left(z_{2}\right)$ onto the unit circle.

We shall denote $\left(z, Z ; \mathfrak{B}^{2}\right)=\left(Z, z ; \mathfrak{B}^{2}\right)$ as the $\mathcal{C}\left(\mathfrak{B}^{2}\right)$-distance between the points $z$ and $Z$. $\left(z, Z ; \mathfrak{B}^{2}\right)$ is invariant with respect to conformal transformations. If $\mathbb{E}^{2}$ is the unit circle with center at $Z$, then $\left(z, Z ; \mathbb{\mho}^{2}\right)$ is the Euclidean distance between $z$ and $Z$. We note that 


$$
\left(z, Z ; \mathfrak{B}^{2}\right)=\frac{\exp \left[z, Z ; \mathfrak{B}^{2}\right]-1}{\exp \left[z, Z ; \mathfrak{B}^{2}\right]+1}
$$

where $\left[z, Z ; \mathfrak{B}^{2}\right]$ is the non-Euclidian distance between the points $z$ and $Z$ measured with the invariant metric defined in $\mathfrak{B}^{2}$. Thus

$$
\log \left|f_{2}\left(z_{1}, z_{2}\right)\right|-\varepsilon_{2}\left(z_{1}, z_{2}\right)=\log b\left(z_{1}, f_{2} ; z_{2}\right),
$$

where $b\left(z_{1}, f_{2} ; \mathfrak{B}^{2}\left(z_{2}\right)\right)=\prod_{m=1}^{N_{2}\left(z_{2}\right)}\left[\left(z_{1}, \alpha_{2 m}\left(z_{2}\right) ; \mathfrak{B}^{2}\left(z_{2}\right)\right)\right]^{\operatorname{sg}\left(\alpha_{2 n}\left(z_{2}\right)\right)}$, is a quotient, $b=b_{1} / b_{2}$, of generalized Blaschke products $b_{k}, k=1,2\left(b_{1}\right.$ vanishes at zero points, $b_{2}$ at poles of $f_{2}$ in $\mathfrak{B}^{2}\left(z_{2}\right)$ ). Functions $b$ will be denoted as Blaschke functions. (See p. 415 and N. 1, p. 137, B.3.)

It is clear that the integral

$$
\begin{aligned}
\int_{\varsigma_{\mathrm{P}}^{2}} & \sum_{n=1}^{N_{1}\left(z_{2}\right)} \operatorname{sg}\left(\alpha_{1 n}\left(z_{2}\right)\right) \\
& \sum_{m=1}^{N_{2(z 2)}} \operatorname{sg}\left(\alpha_{2 m}\left(z_{2}\right)\right) \log \left|\frac{w\left(\alpha_{1 n}\left(z_{2}\right) ; z_{2}\right)-w\left(\alpha_{2 m}\left(z_{2}\right) ; z_{2}\right)}{1-w\left(\alpha_{1 n}\left(z_{2}\right) ; z_{2}\right) w\left(\alpha_{2 m}\left(z_{2}\right) ; z_{2}\right)}\right| d \omega_{2}
\end{aligned}
$$

exists. Indeed, for $z_{2} \in \mathfrak{S}_{\mathrm{P}}^{2}$,

$$
0<a \leqq\left|\frac{w\left(\alpha_{1 n}\left(z_{2}\right) ; z_{2}\right)-w\left(\alpha_{2 m}\left(z_{2}\right) ; z_{2}\right)}{1-w\left(\alpha_{1 n}\left(z_{2}\right) ; z_{2}\right) \overline{w\left(\alpha_{2 m}\left(z_{2}\right) ; z_{2}\right)}}\right|<1,
$$

since according to Hypothesis 2.IVa the intersection points of zero and pole surfaces of $f_{1}$ and $f_{2}$ lie outside $\left[\mathrm{SP}^{2}\left(z_{2}\right), z_{2} \in \mathfrak{S}_{\mathrm{P}}^{2}\right]$. If we approach to values of $z_{2}$ for which $w\left(\alpha_{k v}\left(z_{2}\right), z_{2}\right) \rightarrow 1$,

$$
\left|\frac{w\left(\alpha_{1 n}\left(z_{2}\right) ; z_{2}\right)-w\left(\alpha_{2 m}\left(z_{2}\right) ; z_{2}\right)}{1-w\left(\alpha_{1 n}\left(z_{2}\right) ; z_{2}\right) \overline{w\left(\alpha_{2 m}\left(z_{2}\right) ; z_{2}\right)}}\right| \rightarrow 1 .
$$

Thus we see that for $z_{2} \in \mathfrak{S}_{\mathrm{P}}^{2}$,

$$
0 \leqq\left|\log \left(\alpha_{1 n}\left(z_{2}\right), \alpha_{2 m}\left(z_{2}\right) ; \mathfrak{B}^{2}\left(z_{2}\right)\right)\right| \leqq|\log a|
$$

uniformly in $z_{2}$ as it can be easily shown by classical arguments. (Concerning $a$, see Hypothesis 4.II.)

Since according to our hypotheses, see Theorem 4.1 , for the number, $N_{k}\left(z_{2}\right)$, of zeros and poles $\alpha_{k n}\left(z_{2}\right)$ of $f_{k}\left(z_{1}, z_{2}\right)$ we have $N_{k}\left(z_{2}\right) \leqq \tilde{N}<\infty$, the integral (7) exists. Thus $\mathcal{X}_{1}(\mathrm{P})=(\pi / 2) \int_{\Phi_{\mathrm{P}}{ }^{2}} \log A\left(z_{2}\right) d \omega_{2}$ where $A\left(z_{2}\right)$ is the product of $\mathcal{C}\left(\mathfrak{B}^{2}\left(z_{2}\right)\right)$-distances between the poles of $f_{1}$ and $f_{2}$ and their zeros, divided by the products of $\mathcal{C}\left(\mathfrak{B}^{2}\left(z_{2}\right)\right)$-distances between the poles of $f_{k}$ and zeros of $f_{3-k}, k=1,2$; $f_{k}=f_{k}\left(z_{1}, z_{2}\right), z_{2}=$ const.

$A\left(z_{2}\right)$ can also be interpreted as the product of Blaschke functions

$$
\prod_{n=1}^{N_{1}(z 2)}\left[b\left(\alpha_{1 n}\left(z_{2}\right), f_{2} ; \mathfrak{B}^{2}\left(z_{2}\right)\right)\right]^{\mathrm{sg}}\left(\alpha_{1 n}\left(z_{2}\right)\right)
$$


where $\alpha_{1 n}\left(z_{2}\right)$ are zero and poles of $f_{1}\left(z_{1}, z_{2}\right), z_{1} \in \mathfrak{B}^{2}\left(z_{2}\right)$.

In the present paper we consider functions $f_{k}, k=1,2$, which for every fixed $z_{2},\left|z_{2}\right| \leqq 1$, possess finitely many, say $N\left(z_{2}\right)$, zeros and $N\left(z_{2}\right) \leqq \tilde{N}$, where $\tilde{N}<\infty$, is independent of $z_{2}$. The methods applied here can be easily generalized to the case where $\lim _{r_{2} \rightarrow 1} N\left(r_{2} \exp \left(i \phi_{2}^{(\nu)}\right)\right)=\infty$, for finitely many $\phi_{2}^{(\nu)}$ or even (under some additional assumption) in the case where $\phi_{2}^{(\nu)}$ have accumulation points. See also N.1, p. 137, B.3, etc.

We proceed now to the interpretation of the second term in (4). For every $z_{2}$ for which the zero or pole surface of $f_{2}$ does not intersect $\mathfrak{b}^{1}\left(z_{2}\right)$ (see (2.2)), $\varepsilon_{2}\left(z_{1}, z_{2}\right)-e_{2}\left(z_{1}, z_{2}\right)$ is a regular harmonic function of $x_{1}, y_{1}$ in $\mathfrak{B}^{2}\left(z_{2}\right)$. Therefore

$$
\varepsilon_{2}-e_{2}
$$

$$
=\frac{1}{2 \pi} \int_{\theta=0}^{2 \pi}\left[\varepsilon_{2}^{*}\left(\lambda, z_{2}\right)-e_{2}^{*}\left(\lambda, z_{2}\right)\right]\left[\frac{e^{i \theta}}{e^{i \theta}-w\left(z_{1}, z_{2}\right)}+\frac{\left.\overline{w\left(z_{1}, z_{2}\right.}\right)}{e^{-i \theta}-\overline{w\left(z_{1}, z_{2}\right)}}\right] d \theta
$$

where $\exp (i \theta)=w^{*}\left(\lambda, z_{2}\right)=w\left(h\left(\lambda, z_{2}\right), z_{2}\right)$, and $w\left(z_{1}, z_{2}\right)$ is the function which maps $\mathfrak{B}^{2}\left(z_{2}\right)$ onto the unit circle. (See p. 416.) For every fixed $\lambda$

$$
\begin{aligned}
\mathcal{E}_{2}^{*}-e_{2}^{*} & =\sum_{\nu=1}^{M_{2}(\lambda)} \operatorname{sg}\left(\beta_{2 \nu}(\lambda)\right) \log \left|\frac{z_{2}-\beta_{2 \nu}(\lambda)}{1-z_{2} \overline{\beta_{2 \nu}(\lambda)}}\right| \\
& =\log \left\{\prod_{\nu=1}^{M_{2}(\lambda)}\left(\left(z_{2}, \beta_{2 \nu}(\lambda)\right)^{\mathbf{s g}\left(\beta_{2 \nu}(\lambda)\right)}\right)\right\}
\end{aligned}
$$

where $\left(z_{2}, Z_{2}\right)=\left(z_{2}, Z_{2} ; \Im^{2}\right)=\left|\left(z_{2}-Z_{2}\right) /\left(1-z_{2} \bar{Z}_{2}\right)\right|$ denotes the $\mathcal{C}\left(\Im^{2}\right)$-distance between $z_{2}$ and $Z_{2}$, and $M_{2}(\lambda)$ the number of zeros and poles of $f_{2}^{*}\left(\lambda, z_{2}\right)$ in $\left|z_{2}\right|<1$. Substituting (9) into (8) (multiplying by $\pi / 2$ and integrating over $\mathfrak{S}_{\mathrm{P}}^{2}$ ) we obtain

$$
\begin{aligned}
\mathcal{X}_{2}=-\frac{i}{4} \int_{\mathscr{S P}^{2}}\left\{\sum_{\mu=1}^{N_{1}\left(z_{2}\right)} \operatorname{sg}\left(\alpha_{1 \mu}\left(z_{2}\right)\right)\right. \\
\quad \int_{\lambda=0}^{2 \pi}\left[\sum_{\nu=1}^{M_{2}(\lambda)} \operatorname{sg}\left(\beta_{2 v}(\lambda)\right) \log \left|\frac{z_{2}-\beta_{2 \nu}(\lambda)}{1-z_{2} \overline{\beta_{2 \nu}(\lambda)}}\right|\right] \\
\left.\quad \times P\left(w\left(\alpha_{1 \mu}\left(z_{2}\right)\right), w^{*}\left(\lambda, z_{2}\right)\right)\right\} \frac{\partial w^{*}\left(\lambda, z_{2}\right)}{\partial \lambda} \frac{d \lambda d \omega_{2}}{w^{*}\left(\lambda, z_{2}\right)}
\end{aligned}
$$

where

$$
\begin{aligned}
P\left(w\left(\alpha_{1 \mu}\left(z_{2}\right)\right), w^{*}\left(\lambda, z_{2}\right)\right) & =\frac{w^{*}\left(\lambda, z_{2}\right)}{w^{*}\left(\lambda, z_{2}\right)-w\left(\alpha_{1 \mu}\left(z_{2}\right), z_{2}\right)}+\frac{\frac{\overline{w\left(\alpha_{1 \mu}\left(z_{2}\right), z_{2}\right)}}{w w^{*}\left(\lambda, z_{2}\right)}-\overline{w\left(\alpha_{1 \mu}\left(z_{2}\right), z_{2}\right)}}{} \\
& =\frac{1-\left|w\left(\alpha_{1 \mu}\left(z_{2}\right), z_{2}\right)\right|^{2}}{1+\left|w\left(\alpha_{1 \mu}\left(z_{2}\right), z_{2}\right)\right|^{2}-2\left|w\left(\alpha_{1 \mu}\left(z_{2}\right), z_{2}\right)\right| \cos \Phi},
\end{aligned}
$$




$$
\Phi=\operatorname{arc} w^{*}\left(\lambda, z_{2}\right)-\operatorname{arc} w\left(\alpha_{1 \mu}\left(z_{2}\right), z_{2}\right) .
$$

The integrand of (10) can become infinite only if $w^{*}\left(\lambda, z_{2}\right)=w\left(\alpha_{1 \mu}\left(z_{2}\right), z_{2}\right)$ (i.e., at a point where a zero or pole surface of $f_{1}$ intersects $\mathfrak{b}^{3}$ ) or if $z_{2}=\beta_{2 v}(\lambda)$.

1. Let $z_{1}=h\left(\lambda, \zeta_{2}(\lambda)\right), z_{2}=\zeta_{2}(\lambda)$ be a segment of the intersection line of $\log \left|f_{1}\left(z_{1}, z_{2}\right)\right|= \pm \infty$ with $\left[z_{1}=h\left(\lambda, z_{2}\right),\left|z_{2}\right| \leqq 1\right]$. For every fixed $\lambda$ in the neighborhood of $z_{2}=\zeta_{2}(\lambda), \bar{z}_{2}=\bar{\zeta}_{2}(\lambda)$

$$
\begin{aligned}
& w\left(h\left(\lambda, z_{2}\right) ; z_{2}\right)-w\left(\alpha_{1 \mu}\left(z_{2}\right) ; z_{2}\right) \\
& \quad=A_{10}(\lambda)\left(z_{2}-\zeta_{2}(\lambda)\right)+A_{01}(\lambda)\left(\bar{z}_{2}-\overline{\zeta_{2}(\lambda)}\right)+\cdots .
\end{aligned}
$$

We make now the additional Hypothesis 6.I. For $z_{2} \in \mathfrak{S}_{\mathrm{P}}^{2}$,

$$
\left|A_{10}(\lambda)\right| \neq\left|A_{01}(\lambda)\right| \text {. }
$$

If we introduce polar coordinates $z_{2}-\zeta_{2}(\lambda)=r_{2} e^{i \phi_{2}}, d \omega_{2}=r_{2} d r_{2} d \phi_{2}$, then for a fixed $\lambda$

$$
\begin{aligned}
A_{10}(\lambda)\left(z_{2}-\zeta_{2}(\lambda)\right)+A_{01}(\lambda)\left(\bar{z}_{2}-\overline{\zeta_{2}(\lambda)}\right) & +\cdots \\
& =r_{2}\left[A_{10}(\lambda) e^{i \phi_{2}}+A_{01}(\lambda) e^{-i \phi_{2}}\right]+\cdots
\end{aligned}
$$

and

$$
\frac{d \omega_{2}}{w\left(h\left(\lambda, z_{2}\right), z_{2}\right)-w\left(\alpha_{1 \mu}\left(z_{2}\right), z_{2}\right)}=\frac{d r_{2} d \phi_{2}}{\left[A_{10}(\lambda) e^{i \phi_{2}}+A_{01}(\lambda) e^{-i \phi_{2}}\right]+r_{2}[\cdots]} .
$$

Consequently (12) remains bounded also in the neighborhood of intersection points of $\mathfrak{b}^{3}$ with the zero and pole surfaces of $f_{1}$ for $z_{2} \in \mathfrak{S}_{P}^{2}$.

2. For every fixed $\lambda$,

$$
\log \left|\frac{z_{2}-\beta_{2 v}(\lambda)}{1-z_{2} \overline{\beta_{2 v}(\lambda)}}\right|
$$

becomes infinite at finitely many points, say $\beta_{2 \nu}(\lambda), \nu=1,2, \cdots, M_{2}(\lambda)$. If $\beta_{2 \nu}(\lambda)$ lies inside $\mathfrak{(}^{2}$ obviously

$$
\int_{\left|z_{2}-\beta_{2 v}(\lambda)\right| \leqq \rho_{2}} \log \left|\frac{z_{2}-\beta_{2 \nu}(\lambda)}{1-z_{2} \overline{\beta_{2 \nu}(\lambda)}}\right| d r_{2} d \phi_{2}
$$

is bounded. (If $\beta_{2 v}(\lambda)$ lies on the boundary of $\mathbb{E}^{2}$ the integrand vanishes.) Thus $\mathcal{X}_{2}$ represents the following integral: For every value $\lambda, 0 \leqq \lambda \leqq 2 \pi, z_{2} \in \subseteq_{\mathrm{P}}^{2}$, we form the sum of the products of the logarithm of the Blaschke function

$$
b\left(z_{2}, f_{2}^{*}\left(\lambda, z_{2}\right) ; \mho^{2}\right)=\prod_{\nu}\left|\frac{z_{2}-\beta_{2 v}(\lambda)}{1-z_{2} \overline{\beta_{2 v}(\lambda)}}\right|^{\mathrm{sg}\left(\beta\left(\lambda_{2 v}\right)\right)}
$$

by

$\operatorname{sg}\left(\alpha_{1 \mu}\left(z_{2}\right)\right) \Phi\left(w\left(\alpha_{1 \mu}\left(z_{2}\right), z_{2}\right), w^{*}\left(\lambda, z_{2}\right)\right)$. 
Here $\beta_{2 v}(\lambda)$ are zeros and poles of $f_{2}^{*}\left(\lambda, z_{2}\right)$ in $\left|z_{2}\right| \leqq 1, \Phi$ is the Poisson kernel, and $\alpha_{1 \mu}\left(z_{2}\right)$ are the zeros and poles of $f_{1}\left(z_{1}, z_{2}\right)$ in $\mathfrak{B}^{2}\left(z_{2}\right) . \mathcal{X}_{2}$ is the integral of this sum multiplied by the weighting function

$$
\frac{1}{4 i w^{*}\left(\lambda, z_{2}\right)} \frac{\partial w^{*}\left(\lambda, z_{2}\right)}{\partial \lambda}
$$

over $\left[0 \leqq \lambda \leqq 2 \pi, z_{2} \in \Im_{P}^{2}\right]$.

7. The kernel function of the extended class $\varepsilon(\mathfrak{B})$. In $[\mathrm{B} .4,5,6,10]$ as well as in $\S \S 2-6$ of the present paper it has been shown that the extended class represents a useful tool in the study of various problems of the theory of functions of two complex variables. It is therefore of interest to investigate further properties of these functions. In the present section we shall consider functions of the extended class in domains described in $\$ 2$. These domains are bounded by two segments of analytic hypersurfaces $\mathfrak{a}^{3}$ and $\mathfrak{b}^{3}$ (see (2.4) and (2.5)). In addition to the hypotheses of $\$ 2$, we are making in the present section the following assumption 7.I about the structure of the domains $\mathfrak{B}$.

By a transformation $\left({ }^{12}\right)$

$$
z_{1}^{*}=w\left(z_{1}, z_{2}\right)=w\left(z_{1} ; z_{2}, \bar{z}_{2}\right), \quad z_{2}^{*}=z_{2},
$$

where $w$ is a conveniently chosen function which is analytic in $z_{1}$ (but not necessarily in $z_{2}$ ) and which satisfies the conditions

$$
w\left[h\left(0, z_{2}\right), z_{2}\right]=w\left[h\left(2 \pi, z_{2}\right), z_{2}\right]=1, \quad w\left(0, z_{2}\right)=0 .
$$

$\mathfrak{B}$ is mapped onto the bicylinder $\mathfrak{B}^{*}=\left[\left|z_{1}^{*}\right|<1,\left|z_{2}\right|<1\right]$. Every segment of the analytic surface $\left[z_{1}=h_{1}\left(e^{i \lambda}, z_{2}\right),\left|z_{2}\right| \leqq 1\right], \lambda=$ const., goes over into the segment

$$
\begin{aligned}
& {\left[\operatorname{arc} z_{1}^{*}=\operatorname{arc} W\left(e^{i \lambda}, z_{2}\right),\left|z_{1}^{*}\right|=1,\left|z_{2}\right| \leqq 1\right],} \\
& W\left(e^{i \lambda}, z_{2}\right)=W\left(e^{i \lambda}, z_{2}, \bar{z}_{2}\right) \equiv w\left[h_{1}\left(e^{i \lambda}, z_{2}\right), z_{2}, \bar{z}_{2}\right]
\end{aligned}
$$

We shall assume in this section that the domain $\mathfrak{B}$ has the property that

$$
0<a \leqq \frac{\operatorname{arc} W\left(e^{i \lambda}, z_{2}\right)-\operatorname{arc} W\left(e^{i \mu}, z_{2}\right)}{\lambda-\mu} \leqq A<\infty
$$

where $a$ and $A$ are conveniently chosen constants. (Hypothesis 7.I.)

Notation. The functions $H^{\dagger}\left(x_{1}, y_{1}, x_{2}, y_{2}\right)$ of $\varepsilon(\mathfrak{B})$ which are continuous on the distinguished boundary surface and satisfy the condition

$$
H^{\dagger}\left(0,0, x_{2}, y_{2}\right)=0
$$

will be said to belong to $\varepsilon_{0}(\mathfrak{B})$.

THEOREM 7.1. There exists a system of real functions $\phi_{p}^{\dagger}\left(x_{1}, y_{1}, x_{2}, y_{2}\right)$

(12) Since $z_{2}^{*}=z_{2}$, we omit the asterisk at $z_{2}^{*}$. 
$\equiv T_{p}\left(z_{1}, z_{2}\right), p=1,2, \cdots$, of the class $\varepsilon_{0}(\mathfrak{B})$ which are orthonormal in the sense that

$$
\begin{aligned}
\int_{\mathscr{B}}\left[\frac{\partial \phi_{\nu}^{\dagger}}{\partial x_{1}} \frac{\partial \phi_{\mu}^{\dagger}}{\partial x_{1}}+\frac{\partial \phi_{\nu}^{\dagger}}{\partial y_{1}} \frac{\partial \phi_{\mu}^{\dagger}}{\partial y_{1}}\right] d \omega & =4 \operatorname{Re}\left[\int_{\mathscr{B}} \frac{\partial T_{\nu}}{\partial z_{1}} \frac{\partial T_{\mu}}{\partial \bar{z}_{1}} d \omega\right] \\
& =\delta_{\nu \mu}=\left\{\begin{array}{l}
1 \text { if } \nu=\mu, \\
0 \text { if } \nu \neq \mu,
\end{array}\right.
\end{aligned}
$$

and possess the following properties:

$1^{0}$. The system $\left\{T_{p}\right\}$ is (uniformly) complete on the distinguished boundary surface $\mathfrak{D}^{2}$, i.e., possesses the property that every continuous function defined on $\mathfrak{D}^{2}$ can be uniformly approximated by a linear combination

$$
\sum_{p=1}^{N} a_{p} T_{p}\left(z_{1}, z_{2}\right),\left(z_{1}, z_{2}\right) \in \mathfrak{D}^{2}, N<\infty, \quad\left(a_{p} \equiv a_{p}^{(N)} \text { are constants }\right) .
$$

$2^{0}$. The kernel function of the system $\left\{T_{p}\right\}$ converges in $\mathfrak{B}$, i.e., we have

$$
\sum_{\nu=1}^{\infty}\left[\phi_{\nu}^{\dagger}\left(x_{1}, y_{1} ; x_{2}, y_{2}\right)\right]^{2}<\infty \quad \text { for }\left(x_{1}, y_{1}, x_{2}, y_{2}\right) \in \mathfrak{B} \text {. }
$$

Proof. The proof of the theorem follows in the usual way (see also pp. 451-452) from the following

Lемма. If $\mathcal{F}=\sum_{\nu=1}^{m} \alpha_{\nu} T_{\nu}$ satisfies the condition

$$
\left(\frac{\partial \mathcal{F}}{\partial z_{1}}\right)_{z_{1=\zeta_{1}, z_{2}=\zeta_{2}}}=1, \quad\left(\zeta_{1}, \zeta_{2}\right) \in \mathfrak{B}
$$

then

$$
\int_{\mathfrak{B}}\left|\frac{\partial \mathcal{F}}{\partial z_{1}}\right|^{2} d \omega \geqq C\left(\zeta_{1}, \zeta_{2}\right)>0
$$

where $C\left(\zeta_{1}, \zeta_{2}\right)$ is a fixed positive constant which depends only on $\mathfrak{B}$ and $\zeta_{1}, \zeta_{2}$.

Proof. We consider at first the case of $\mathfrak{B}=\mathfrak{C}=\left[\left|z_{1}\right|<1,\left|z_{2}\right|<1\right]$. In this case the real and imaginary parts of the set

$$
z_{1}^{m}, z_{1}^{m} z_{2}^{n}, z_{1}^{m} \bar{z}_{2}^{n}, \quad m=0,1,2,3, \cdots, n=1,2,3, \cdots,
$$

form a complete system. Using polar coordinates, i.e., writing $z_{k}=x_{k}+i y_{k}$ $=r_{k}\left(\cos \phi_{k}+i \sin \phi_{k}\right), k=1,2$, the real and imaginary parts of functions of the system (9) can be written in the form $\left({ }^{13}\right)$

(13) We note that in (10) and in the following we use the double index $\{m, n\}$ instead of the index $[p], p=1,2, \cdots$. The functions of the set $(10)$ are assumed to be ordered in such a way that $\{1,0\},\{2,0\},\{1,1\},\{1,-1\}, \cdots$, correspond to $[1],[2],[3],[4], \cdots$ We omit the terms corresponding to $m=0$, since the derivatives of these terms with respect to $z_{1}$ vanish. 


$$
\stackrel{m}{r_{1}} \cos m \phi_{1}, r_{2}^{m} \sin m \phi_{1}, r_{1} r_{2}^{|n|} \cos \left(m \phi_{1}+n \phi_{2}\right), r_{1} r_{2}^{|n|} \sin \left(m \phi_{1}+n \phi_{2}\right),
$$

$m=1,2, \cdots ; n=1,-1,2,-2, \cdots$. The functions (10) are orthogonal to each other in the sense (5) (with $\mathfrak{B}=\mathbb{C}$ ). Dividing them by conveniently chosen constants we obtain a system which is orthonormal in $\mathfrak{5}$.

Using the well-known development of a (real) continuous function $F$ defined on the distinguished boundary surface [see e.g. [B.9], [B.-Marcinkiewicz 1], [Bers 1]], we conclude that every $F$ can be developed in $\bar{\complement}$ in a series

$$
\begin{aligned}
F= & \sum_{m=1}^{\infty}\left\{\alpha_{m 0}\left(z_{1}^{m}+\bar{z}_{1}^{m}\right)-i \beta_{m 0}\left(z_{1}^{m}-\bar{z}_{1}^{m}\right)\right. \\
& +\sum_{n=1}^{\infty}\left[\alpha_{m n}\left(z_{1}^{m} z_{2}^{n}+\bar{z}_{1}^{m} \bar{z}_{2}^{n}\right)-i \beta_{m n}\left(z_{1}^{m} z_{2}^{n}-\bar{z}_{1}^{m} \bar{z}_{2}^{n}\right)\right. \\
& \left.\left.+\gamma_{m n}\left(z_{1}^{m} \bar{z}_{2}^{n}+\bar{z}_{1}^{m} z_{2}^{n}\right)-i \delta_{m n}\left(z_{1}^{m} \bar{z}_{2}^{n}-\bar{z}_{1}^{m} z_{2}^{n}\right)\right]\right\} .
\end{aligned}
$$

The condition ( 7 ) with $\zeta_{1}=0, \zeta_{2}=0$ yields $\alpha_{10}=1, \beta_{10}=0$. On the other hand $F_{1}=z_{1}$ is orthogonal in the sense (5) to each of the functions $\bar{z}_{1}^{m}, m=2,3, \cdots$, $\bar{z}_{1}^{m} \bar{z}_{2}^{n}, \bar{z}_{1}^{m} z_{2}^{n}, m=1,2,3, \cdots, n=1,2,3, \cdots$, and consequently

$$
\begin{gathered}
\int_{\mathfrak{S}}\left|\frac{\partial F}{\partial z_{1}}\right|^{2} d \omega=\int_{\mathfrak{S}} d \omega+\int_{\mathfrak{S}}|S|^{2} d \omega \geqq \int_{\mathfrak{C}} d \omega=\pi^{2}=C, \\
S=\sum_{m=2}^{\infty} m\left(\alpha_{m 0}-i \beta_{m 0}\right) z_{1}^{m-1}+\sum_{m=1}^{\infty} \sum_{n=1}^{\infty}\left[m\left(\alpha_{m n}-i \beta_{m n}\right) z_{1}^{m-1} z_{2}^{n}\right. \\
\left.\quad+m\left(\gamma_{m n}-i \delta_{m n}\right) z_{1}^{m-1} \bar{z}_{2}^{n}\right]
\end{gathered}
$$

and $C$ is independent of $F$ (satisfying (7) with $\zeta_{1}=\zeta_{2}=0$ ). If instead of the origin we choose an arbitrary interior point $\left(\zeta_{1}, \zeta_{2}\right)$ of $(\mathfrak{S}$, an analogous consideration leads to an inequality (12) with $C=\pi^{2}\left(1-\left|\zeta_{1}\right|^{2}\right)\left(1-\left|\zeta_{2}\right|^{2}\right)$.

We proceed now to prove an inequality analogous to (8) for general domains $\mathfrak{B}$, as described in $\$ 2$ and satisfying 7.I. By the transformation (1) satisfying (2) $\mathfrak{B}$ is mapped onto the bicylinder $\mathfrak{B}^{*}=\left[\left|z_{1}^{*}\right|<1,\left|z_{2}\right|<1\right]$. Every segment of the analytic surface $\left[z_{1}^{*}=h_{1}\left(e^{i \lambda}, z_{2}\right),\left|z_{2}\right| \leqq 1\right], \lambda=$ const., goes over into a segment

$$
\text { [ } \left.\operatorname{arc} z_{1}^{*}=\operatorname{arc} W\left(e^{i \lambda}, z_{2}\right),\left|z_{1}^{*}\right|=1,\left|z_{2}\right| \leqq 1\right], W\left(e^{i \lambda}, z_{2}\right) \equiv w\left(h_{1}\left(e^{i \lambda}, z_{2}\right), z_{2}\right] \text {. }
$$

The class of functions $\mathcal{E}(\mathfrak{B})$ goes into the class $\mathcal{S}\left(\mathfrak{B}^{*}\right)$. Every function $K_{p}\left(z_{1}, z_{2}\right)$ of the system (10) assumes continuous values, say $K_{p}\left(e^{i \lambda}, e^{i \psi}\right)$ on $\left[\left|z_{1}\right|=1\right.$, $\left.\left|z_{2}\right|=1\right]$. Let now $\tau_{n}\left(z_{1}^{*}, z_{2}\right) \in \mathcal{S}\left(\mathfrak{B}^{*}\right)$ be so determined that

$$
\begin{array}{r}
\tau_{p}\left[w\left(h_{1}\left(e^{i \lambda}, e^{i \phi_{2}}\right), e^{i \phi_{2}}\right), e^{i \phi_{2}}\right]=K_{p}\left(e^{i \lambda}, e^{i \phi_{2}}\right), \quad h_{1}\left(e^{i \lambda}, e^{i \phi_{2}}\right) \equiv h\left(\lambda, e^{i \phi_{2}}\right), \\
0 \leqq \lambda \leqq 2 \pi, 0 \leqq \phi_{2} \leqq 2 \pi, p=1,2, \cdots .
\end{array}
$$


In the following, the lamina $\left[z_{1}^{*}=\exp \left(i w\left(h\left(\lambda, z_{2}\right), z_{2}\right)\right),\left|z_{2}\right|<1, \lambda\right.$ constant $]$ will be denoted by $\mathbb{8}^{2}(\lambda)$.

Through every point $\left\{\exp (i \mu), \zeta_{2}\right\}$ of $\left[\left|z_{1}^{*}\right|=1,\left|z_{2}\right| \leqq 1\right]$ goes a lamina $\mathcal{B}^{2}(\lambda)$ where $\lambda$ is the solution of the equation

$$
\exp (i \mu)=w\left[h\left(\lambda, \zeta_{2}\right), \zeta_{2}\right]
$$

Since in every lamina $z^{2}(\lambda)$, the function $\left.\tau_{p}\left[w\left(h\left(\lambda, z_{2}\right)\right), z_{2}\right), z_{2}\right]$ is a harmonic function of $x_{2}, y_{2}$ it follows from (15) that

$$
\tau_{p}\left[w\left(h\left(\mu, z_{2}\right), z_{2}\right), z_{2}\right]=K_{p}\left(e^{i \lambda}, z_{2}\right) \quad \text { for }\left|z_{2}\right|<1
$$

where

$$
\mu \equiv \mu\left(\lambda, z_{2}\right), \quad \mu\left(0, z_{2}\right)=0 .
$$

By orthogonalizing the system $\left\{\tau_{n}\right\}$ we obtain the system $\left\{T_{p}\right\}, p$ $=1,2, \cdots$, of functions of the class $S\left(\mathfrak{B}^{*}\right)$ which are orthonormal in the sense (5). We proceed now to prove the inequality (8) for functions $F$ of the class $S\left(\mathfrak{B}^{*}\right)$ satisfying the condition (7). Since $\left(\zeta_{1}, \zeta_{2}\right) \in \mathfrak{B}^{*},\left|\zeta_{1}\right|<1,\left|\zeta_{2}\right|<1$. Let us form a function

$$
\mathcal{F}_{N}\left(z_{1}^{*}, z_{2}\right)=\sum_{n=1}^{N} \beta_{n} T_{n}\left(z_{1}^{*}, z_{2}\right)
$$

possessing the property that $\mathcal{F}_{N}$ satisfies the condition (7).

As we mentioned before through every point $\left\{e^{i \mu}, \zeta_{2}\right\},\left|\zeta_{2}\right| \leqq 1$, goes the lamina $\varepsilon^{2}(\lambda)$, where $\lambda$ has to be determined from (16). In every segment $\varepsilon^{2}(\lambda)$, $\mathcal{f}_{N}$ assumes the values $\mathcal{f}_{N}\left[\exp \left(i \mu\left(\lambda, z_{2}\right)\right), z_{2}\right] . \mathcal{F}_{N}\left[\exp \left(i \mu\left(\lambda, z_{2}\right)\right), z_{2}\right], \lambda$ const., is a harmonic function of $x_{2}, y_{2}$.

We determine now a function $P_{N}\left(z_{1}, z_{2}\right)$ of the class $\mathcal{E}(\mathfrak{E})$ by requiring that $\left({ }^{14}\right)$

$$
P_{N}\left[\exp \left(\left(i \mu\left(\lambda, \zeta_{2}\right)\right), z_{2}\right]=\mathcal{F}_{N}\left[\exp \left(\left(i \mu\left(\lambda, z_{2}\right)\right), z_{2}\right] .\right.\right.
$$

$P_{N}\left(z_{1}, \zeta_{2}\right)$ and $\mathcal{F}_{N}\left(z_{1}^{*}, \zeta_{2}\right)$ are harmonic functions of $x_{1}, y_{1}$ in $\left|z_{1}\right|<1$, and of $x_{1}^{*}, y_{1}^{*}$ in $\left|z_{1}^{*}\right|<1$. Therefore from $P_{N}\left(e^{i \lambda}, \zeta_{2}\right)=\mathcal{F}_{N}\left(e^{i \lambda}, \zeta_{2}\right)$ follows $P_{N}\left(z_{1}\right.$, $\left.\zeta_{2}\right)=\mathcal{F}_{N}\left(z_{1}^{*}, \zeta_{2}\right),\left|z_{1}\right| \leqq 1$. Therefore, in particular

$$
\left(\frac{\partial P_{N}}{\partial z_{1}}\right)_{z_{1}=\zeta_{1}, z_{2}=\zeta_{2}}=\left(\frac{\partial \mathcal{F}_{N}}{\partial z_{1}^{*}}\right)_{z_{1}^{*}=\zeta_{1}, z_{2}=\zeta_{2}}=1 .
$$

According to (11) and (12), we have

$$
\int_{\mathfrak{G}}\left|\frac{\partial P_{N}}{\partial z_{1}}\right|^{2} d \omega \geqq C\left(\zeta_{1}, \zeta_{2}\right) \equiv \pi^{2}\left(1-\left|\zeta_{1}\right|\right)^{2}\left(1-\left|\zeta_{2}\right|\right)^{2}
$$

(14) We note that the mapping $z_{1}^{*}=z_{1}^{*}\left(z_{1}, z_{2}\right), z_{2}=z_{2}$ defined in $\left[\left|z_{1}\right|=1,\left|z_{2}\right| \leqq 1\right]$ has the property that $z_{1}^{*}\left(z_{1}, \zeta_{2}\right)=z_{1}$. 
Let us now compare

and

$$
J\left(z_{2}\right)=\int_{\left|z_{1}\right|<1}\left|\frac{\partial P_{N}\left(z_{1}^{*}, z_{2}\right)}{\partial z_{1}^{*}}\right|^{2} d \omega_{1}^{*}
$$

$$
\Im^{\dagger}\left(z_{2}\right)=\int_{\left|z_{1}{ }^{*}\right|<1}\left|\frac{\partial \mathcal{F}_{N}\left(z_{1}, z_{2}\right)}{\partial z_{1}}\right|^{2} d \omega_{1}
$$

For $z_{2}=\zeta_{2}$, as we mentioned, $\Im\left(\zeta_{2}\right)=\Im^{\dagger}\left(\zeta_{2}\right)$.

According to a formula of Douglass [D. 1, 2], [Sh. 1], [M.-T. 1],

$$
\begin{aligned}
& \Im\left(z_{2}\right)=\frac{1}{16 \pi} \int_{\phi=0}^{2 \pi} \int_{\theta=0}^{2 \pi}\left|\frac{P_{N}\left(\exp (i \phi), z_{2}\right)-P_{N}\left(\exp (i \theta), z_{2}\right)}{\sin \left[\frac{1}{2}(\phi-\theta)\right]}\right|^{2} d \theta d \phi, \\
& J^{\dagger}\left(z_{2}\right)=\frac{1}{16 \pi} \int_{\phi=0}^{2 \pi} \int_{\theta^{*}=0}^{2 \pi}\left|\frac{\mathcal{F}_{N}\left(\exp \left(i \phi^{*}\right), z_{2}\right)-\mathcal{F}_{N}\left(\exp \left(i \theta^{*}\right), z_{2}\right)}{\sin \left[\frac{1}{2}\left(\phi^{*}-\theta^{*}\right)\right]}\right|^{2} d \theta^{*} d \phi^{*} .
\end{aligned}
$$

Here $\phi^{*}\left(z_{2}\right)=\operatorname{arc} z_{1}^{*}=\operatorname{arc}\left[w\left(h\left(e^{i \phi}, z_{2}\right), z_{2}\right)\right]$ and the same formula holds for $\theta^{*}\left(z_{2}\right)$ as a function of $\theta$. Since by (19), $P_{N}\left(e^{i \phi}, z_{2}\right)=\mathcal{F}_{N}\left(e^{i \phi^{*}}, z_{2}\right)$,

$$
\begin{aligned}
J^{\dagger}\left(z_{2}\right) & =\frac{1}{16 \pi} \int_{\phi^{*}=0}^{2 \pi} \int_{\theta=0}^{2 \pi}\left|\frac{P_{N}\left(\exp (i \phi), z_{2}\right)-P_{N}\left(\exp (i \theta), z_{2}\right)}{\sin \left(\frac{1}{2}(\phi-\theta)\right)}\right|^{2} d \phi d \theta \\
& \times \frac{\sin \left(\frac{1}{2}(\phi-\theta)\right)}{\sin \left(\frac{1}{2}\left(\phi^{*}-\theta^{*}\right)\right)} \frac{d \phi^{*}}{d \phi} \frac{d \theta^{*}}{d \theta} .
\end{aligned}
$$

For every $\left|z_{2}\right| \leqq 1$, according to (3)

$$
0<a^{2} \leqq \frac{d \phi^{*}}{d \phi} \frac{d \theta^{*}}{d \theta} \leqq A^{2}<\infty .
$$

In order to obtain bounds for $\left|\sin ((\phi-\theta) / 2) / \sin \left(\left(\phi^{*}-\theta^{*}\right) / 2\right)\right|^{2}$ we distinguish two cases (a) and (b), assuming

$$
0 \leqq|\phi-\theta| \leqq \frac{\pi}{2 A} \quad \text { or } \quad\left(27^{\prime} a\right) \quad 0 \leqq 2 \pi-|\phi-\theta| \leqq \frac{\pi}{2 A}
$$

and

$$
\frac{\pi}{2 A}<|\phi-\theta|<2 \pi\left(1-\frac{1}{4 A}\right) .
$$

In the case (a), we have

$$
\left|\frac{\sin (s / 2)}{\sin \left(s^{*} / 2\right)}\right|^{2} \equiv\left|\frac{2 \sin (s / 2) / s}{2 \sin \left(s^{*} / 2\right) / s^{*}}\right|^{2}\left|\frac{s}{s^{*}}\right|^{2} .
$$

Here $s=|\phi-\theta|, s^{*}=\left|\phi^{*}-\theta^{*}\right|$ in the case (27a) and $s=2 \pi-|\phi-\theta|, s^{*}=2 \pi$ $-\left|\phi^{*}-\theta^{*}\right|$ in the case (27'a), respectively. From (3) follows in the case (a) 


$$
0 \leqq s^{*} \leqq \pi / 2
$$

Since $2 / \pi \leqq[(\sin \alpha) / \alpha] \leqq 1$ for $|\alpha|<\pi / 2$, in the case (a) we have

$$
\left(\frac{2}{\pi}\right)^{2} a^{2} \leqq\left|\frac{\sin (s / 2) / s}{\sin \left(s^{*} / 2\right) / s^{*}}\right|^{2}\left|\frac{s}{s^{*}}\right|^{2} \leqq A^{2}\left(\frac{\pi}{2}\right)^{2} .
$$

In the case (b) according to (26)

$$
\frac{\pi a}{2 A} \leqq\left|\phi^{*}-\theta^{*}\right| \leqq 2 \pi-\frac{\pi a}{2 A}
$$

and therefore

$$
[\sin (\pi a / 4 A)]^{2} \leqq\left|\frac{\sin (s / 2)}{\sin \left(s^{*} / 2\right)}\right|^{2} \leqq \frac{1}{[\sin (\pi a / 4 A)]^{2}} .
$$

If therefore

$$
b=\min \left[\left(\sin \left(\pi a / 4 A^{2}\right)\right),(2 a / \pi)\right], \quad B=\max [1 / \sin (\pi a / 4 A), \pi A / 2],
$$

we see that

$$
0<b^{2} a^{2} \leqq \frac{J^{*}\left(z_{2}\right)}{\Im\left(z_{2}\right)} \leqq B^{2} A^{2}<\infty .
$$

Using (34), (20), and (13), we see that

$$
\begin{aligned}
\int_{\mathfrak{B}^{*}}\left|\frac{\partial F_{N}}{\partial z_{1}^{*}}\right|^{2} d \omega & =\int_{\left|z_{2}\right|<\left(1-\left|\zeta_{2}\right|\right)} J \dagger\left(z_{2}\right) d \omega_{2} \geqq b^{2} a^{2} \int_{\left|z_{2}\right|<\left(1-\left|\zeta_{2}\right|\right)} J\left(z_{2}\right) d \omega_{2} \\
& =\pi b^{2} a^{2}\left(1-\left|\zeta_{1}\right|\right)^{2}\left(1-\left|\zeta_{2}\right|\right)^{2} .
\end{aligned}
$$

After the inequality (35) for functions $F_{N}^{*}$ satisfying (7) have been derived the proof that the kernel function is finite follows in the usual way. (See [8, pp. $21 \mathrm{ff}$.$] .)$

We determine the $\alpha_{n}, n=1,2, \cdots, N$, so that the expression

$$
4 \int_{B}\left|\sum_{n=1}^{N} \alpha_{n} T_{n}^{\prime}\right|^{2} d \omega=\sum_{n=1}^{N}\left|\alpha_{n}\right|^{2}, \quad T_{n}^{\prime} \equiv \frac{\partial T_{n}}{\partial z_{1}},
$$

becomes minimum $\left({ }^{15}\right)$ under the condition that

$$
\sum_{h=1}^{N} \alpha_{n} T_{n}^{\prime}\left(\zeta_{1}, \zeta_{2}\right)=1
$$

If we write

(15) Since the function $w\left(z_{1}, z_{2}\right)$ in (1) is analytic in $z_{1}$ the value of the integral (36) remains unchanged when we pass from the domain $\mathfrak{B}^{*}$ back to the domain $\mathfrak{B}$. 


$$
\left.\alpha_{n}=\left[\overline{\left(T_{n}^{\prime}\left(\zeta_{1}, \zeta_{2}\right)\right.}+A_{n}\right) / \sum_{\nu=1}^{N}\left|{T^{\prime 2}}^{2}\left(\zeta_{1}, \zeta_{2}\right)\right|^{2}\right],
$$

the condition (37) assumes the form

$$
\sum_{n=1}^{N} A_{\nu} T_{n}^{\prime}\left(\zeta_{1}, \zeta_{2}\right)=0
$$

and

$$
\sum_{n=1}^{N}\left|\alpha_{n}\right|^{2}=\frac{\sum_{n=1}^{N}\left|\overline{T_{n}^{\prime}\left(\zeta_{1}, \zeta_{2}\right)}+A_{n}\right|^{2}}{\left(\sum_{n=1}^{N}\left|T_{n}^{\prime}\left(\zeta_{1}, \zeta_{2}\right)\right|^{2}\right)^{2}}=\frac{\sum_{n=1}^{N}\left|T_{n}^{\prime}\left(\zeta_{1}, \zeta_{2}\right)\right|^{2}+\sum_{n=1}^{N}\left|A_{n}\right|^{2}}{\left(\sum_{n=1}^{N}\left|T_{n}^{\prime}\left(\zeta_{1}, \zeta_{2}\right)\right|^{2}\right)^{2}}
$$

The minimum of (39) will be attained if and only if $A_{n}=0, n=1,2, \cdots, N$, and equals

$$
\frac{1}{\sum_{n=1}^{N}\left|T_{n}^{\prime}\left(\zeta_{1}, \zeta_{2}\right)\right|^{2}} \geqq 4 C\left(\zeta_{1}, \zeta_{2}\right)>0
$$

by (8). Since the right-hand side is independent of $N$ it follows that

$$
\sum_{n=1}^{\infty}\left|T_{n}^{\prime}\left(\zeta_{1}, \zeta_{2}\right)\right|^{2} \leqq \frac{1}{4 C\left(\zeta_{1}, \zeta_{2}\right)}<\infty
$$

(6) follows from (41) and from the relation

$$
\sum_{n=1}^{N}\left|T_{n}\left(z_{1}, z_{2}\right)\right|^{2}=\int_{\zeta_{1}=0}^{z_{1}} \int_{\zeta_{1}^{*}=0}^{z_{1}} \sum_{n=1}^{N} T_{n}^{\prime}\left(\zeta_{1}, z_{2}\right) \overline{T_{n}^{\prime}\left(\zeta_{1}^{*}, z_{2}\right)} d \zeta_{1} d \zeta_{1}^{*}
$$

which holds for the functions of the class $\varepsilon_{0}(\mathfrak{B})$, see (4).

\section{BIBLIOGRAPHY}

\section{S. BERGMAN}

1. Über eine in gewissen Bereichen gültige Integraldarstellung der Funktionen zweier komplexer Variabler, Math. Zeit. vol. 39 (1934) pp. 76-94, 605-608.

2. Über eine Integraldarstellung von Funktionen zweier komplexer Veränderlichen, Rec. Math. (Mat. Sbornik) N.S. vol. 1 (43) (1936) pp. 851-862.

3. On a generalized Green's function and certain of its applications, Bull. Amer. Math. Soc. vol. 47 (1941) pp. 651-661.

4. Ueber meromorphe Funktionen von zwei komplexen Veränderlichen, Compositio Math. vol. 6 (1939) pp. 305-335.

5. A theorem of Green's type for functions of two complex variables, Proc. Amer. Math. Soc. vol. 4 (1953) pp. 102-109.

6. Geometric and potential-theoretical methods in the theory of functions of several complex 
variables, Proceedings of the International Congress of Mathematicians, 1950, vol. II, 1952, pp. 165-173.

7. Sur les fonctions orthogonales de plusieurs variables complexes avec les applications a la théorie des fonctions analytiques, Interscience, 1941, and Mémorial des Sciences Mathématiques, vol. 106, Paris, 1947.

8. The kernel function and conformal mapping, Mathematical Surveys, no. 5, American Mathematical Society, 1950.

9. Über ausgezeichnete Randfä̈hen in der Theorie der Funktionen von zwei komplexen Veränderlichen, Math. Ann. vol. 104 (1931) pp. 611-636.

10. Kernel function and extended classes in the theory of functions of complex variables, Colloque sur les fonctions de plusieurs variables, Centre Belge de Recherches, Math., Brussels, 1953, pp. 135-157.

11. Über den Wertevorrat einer Funktion von zwei komplexen Veränderlichen, Math. Zeit. vol. 36 (1932) pp. 171-183.

S. Bergman and J. MARCINKIEWicZ

1. Sur les fonctions analytiques de deux variables complexes, Fund. Math. vol. 33 (19391946) pp. 75-94, and Journal of Mathematics and Physics vol. 21 (1942) pp. 125-141.

S. Bergman and M. SCHIFFER

1. Kernel functions and elliptic differential equations in mathematical physics, Monographs in Pure and Applied Mathematics, vol. IV, New York, 1953.

2. Potential-theoretic methods in the theory of functions of two complex variables, Compositio Math. vol. 10 (1952) pp. 213-240.

L. BERS

1. On bounded analytic functions of two complex variables in certain domains with distinguished boundary surfaces, Amer. J. Math. vol. 64 (1942) pp. 514-529.

\section{R. Courant}

1. Über eine Eigenschaft der Abbildungsfunktionen bei konformer Abbildung, Abhandlungen der Kön. Gesellschaft d. Wissenschaften zu Göttingen (1914) pp. 101-109.

2. Bemerkung zu meiner Note "Über eine Eigenschaft der Abbildungsfunktionen bei konformer Abbildung," Abhandlungen der Kön. Gesellschaft d. Wissenschaften zu Göttingen (1922) pp. 69-70.

J. Douglas

1. Solution of the problem of Plateau, Trans. Amer. Math. Soc. vol. 33 (1931) pp. 263-321.

2. Minimal surfaces of higher topological structure, Ann. of Math. vol. 40 (1939) pp. 205298.

A. Gelbart

1. On the growth of properties of a function of two complex variables given by its power series expansion, Trans. Amer. Math. Soc. vol. 49 (1941) pp. 199-210.

M. Morse and C. Tompkins

1. The existence of minimal surfaces of general critical types, Ann. of Math. vol. 40 (1939) pp. 443-472.

\section{R. NEVANLINNA}

1. Le thêorème de Picard-Borel et la thêorie des fonctions méromorphes, Paris, 1929.

T. RAD6́

1. Sur la représentation conforme de domaines variables, Acta Litterarum ac Scientiarum Regiae Universitatis Hungaricae vol. 1 (1923) pp. 180-186.

\section{SHIFFMAN}

1. The Plateau problem for non-relative minima, Ann. of Math. vol. 40 (1939) pp. 834-854.

\section{F. SOMMER}

1. Über die Integralformeln in der Funktionentheorie mehrerer komplexer Veränderlichen, Math. Ann. vol. 125 (1952) pp. 172-182. 
A. WEIL

1. Sur les séries de polynomes de deux variables complexes, C. R. Acad. Sci. Paris vol. 194 (1932) p. 1304.

2. L'integral de Cauchy et les fonctions de plusieurs variables, Math. Ann. vol. 111 (1935) pp. 178-182.

STANFORD UNIVERSITY,

Stanford, Calif. 